\title{
Normal A0-A1 stars with low rotational velocities
}

\section{Abundance determination and classification $\star, \star \star$}

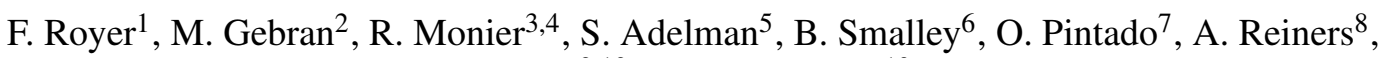 \\ G. Hill ${ }^{9,10}$, and A. Gulliver ${ }^{10}$
}

${ }^{1}$ GEPI/CNRS UMR 8111, Observatoire de Paris - Université Paris Denis Diderot, 5 place Jules Janssen, 92190 Meudon, France e-mail: frederic.royer@obspm.fr

2 Department of Physics and Astronomy, Notre Dame University-Louaize, PO Box 72, Zouk Mikaël, Lebanon

3 LESIA/CNRS UMR 8109, Observatoire de Paris - Université Pierre et Marie Curie - Université Paris Denis Diderot, 5 place Jules Janssen, 92190 Meudon, France

${ }^{4}$ Laboratoire Lagrange, Université de Nice Sophia Antipolis, Parc Valrose, 06100 Nice, France

5 Department of Physics, The Citadel, 171 Moultrie Street, Charleston, SC 29409, USA

6 Astrophysics Group, Keele University, Staffordshire ST5 5BG, UK

7 INSUGEO-CONICET, Tucumán, Argentina

8 Institut für Astrophysik Göttingen, Physik Fakultät, Friedrich-Hund-Platz 1, 37077 Göttingen, Germany

9 18A Stratford St, 1071 Auckland, New Zealand

10 Department of Physics and Astronomy, Brandon University, Brandon, MB R7A 6A9, Canada

Received 27 September 2013 / Accepted 23 December 2013

\section{ABSTRACT}

\begin{abstract}
Context. The study of rotational velocity distributions for normal stars requires an accurate spectral characterization of the objects in order to avoid polluting the results with undetected binary or peculiar stars. This piece of information is a key issue in the understanding of the link between rotation and the presence of chemical peculiarities.

Aims. A sample of 47 low $v \sin i$ A0-A1 stars $\left(v \sin i<65 \mathrm{~km} \mathrm{~s}^{-1}\right)$, initially selected as main-sequence normal stars, are investigated with high-resolution and high signal-to-noise spectroscopic data. The aim is to detect spectroscopic binaries and chemically peculiar stars, and eventually establish a list of confirmed normal stars.

Methods. A detailed abundance analysis and spectral synthesis is performed to derive abundances for 14 chemical species. A hierarchical classification, taking measurement errors into account, is applied to the abundance space and splits the sample into two different groups, identified as the chemically peculiar stars and the normal stars.

Results. We show that about one third of the sample is actually composed of spectroscopic binaries (12 double-lined and five singlelined spectroscopic binaries). The hierarchical classification breaks down the remaining sample into 13 chemically peculiar stars (or uncertain) and 17 normal stars.
\end{abstract}

Key words. stars: early-type - stars: rotation - stars: abundances - stars: chemically peculiar - binaries: spectroscopic

\section{Introduction}

Observations strongly suggested in the 1960s and 1970s that slow rotation is a necessary condition for the presence of peculiarities in the spectra of A-type stars (Preston 1974, and references therein). Since then, the equatorial velocity below which chemically peculiar (CP) stars are observed is found to be around $120 \mathrm{~km} \mathrm{~s}^{-1}$ (Abt \& Moyd 1973; Abt \& Morrell 1995). This observation is supported by theory that links the chemical peculiarities to the diffusion mechanism, occurring when the He II convection zone disappears at equatorial velocities lower than 70-120 $\mathrm{km} \mathrm{s}^{-1}$ (Michaud 1982). Atomic diffusion, under the competitive action of gravitational settling and radiative accelerations, alters the chemical abundances in stellar atmospheres, when mixing motions are weak. In theory, slow rotation should be a sufficient condition for the $\mathrm{CP}$ phenomenon to

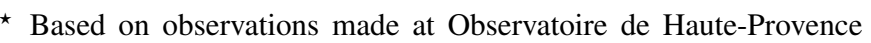
(CNRS), France.

$\star \star$ Tables 1, 2, 4, 5 and Appendices are available in electronic form at http://www . aanda.org
}

appear and Michaud (1980) wondered why a slowly rotating star would be nonpeculiar.

The observational evidence that slow rotation is a sufficient condition for the presence of chemical peculiarities is not as straightforward. Whereas the dichotomy between normal and $\mathrm{CP}$ stars according to rotation rate is rather clear and unanimous in the literature for mid to late A-type stars (Abt \& Moyd 1973; Abt \& Morrell 1995; Royer et al. 2007), this is not the case for early A-type stars, from A0 to A3.

For Abt \& Morrell (1995, hereafter AM), the bimodal shape of the equatorial velocity distribution can be explained by the different rotation rate of normal stars (fast) compared to peculiar and binary stars (slow). They conclude that the observed overlap between both distribution modes for A0-A1 stars is due to the inability to detect marginal CP stars or to evolutionary effects: rotation alone could thus explain the normal or peculiar appearance of an A star's spectrum (Abt 2000; Adelman 2004). However, authors focusing on the rotational velocities of normal stars confirm the excess of slow rotators for normal A0-A1 stars (Dworetsky 1974; Ramella et al. 1989; Royer et al. 2007), 
when removing known $\mathrm{CP}$ and binary stars. This excess of slow rotators is moreover observed at higher masses (Zorec \& Royer 2012).

The nature of these objects remains unclear. There is no doubt that a fraction of them is composed of so far unidentified spectroscopic binaries and/or CP stars; just how many genuine normal stars are slow rotators seems very much open again. Are slowly rotating normal A stars young objects that will become Ap stars and do not show chemical peculiarity yet, as suggested by Abt (2009)? Since the spectroscopic measurement of rotational velocity $(v \sin i)$ is a projection on the line-of-sight, are low $v \sin i$ normal abundance A stars likely to be fast rotators seen at low inclination angle, such as Vega (Gulliver et al. 1994; Hill et al. 2010)? To answer these questions, the low $v \sin i \mathrm{~A} 0-\mathrm{A} 1$ normal stars from Royer et al. (2007, hereafter RZG) need to be investigated with new high-resolution and high signal-to-noise spectroscopic data. The purpose of this article is to perform a detailed abundance analysis and spectral synthesis to detect potential binary or $\mathrm{CP}$ stars and provide a list of confirmed normal low $v \sin i \mathrm{~A} 0-\mathrm{A} 1$ stars. The resulting subsample will be analyzed in greater depth in a following article.

This paper is organized as follows: the sample, its selection and the spectroscopic observations are described in Sect. 2. Section 3 deals with the determination of radial velocities and gives a list of suspected binaries. The atmospheric parameters and rotational velocities are, respectively, derived in Sect. 4 and Sect. 5. Section 6 presents the determination of abundance patterns and Sect. 7 details the classification based on these chemical abundances. The abundance patterns and the rotational velocity distribution are discussed in Sect. 8 and the results are summarized in Sect. 9. Comments on individual stars are given in Appendix A.

\section{Data sample}

\subsection{Target selection}

RZG built a sample of main-sequence A-type stars with homogenized $v \sin i$ using values from AM and Royer et al. (2002a,b). Chemically peculiar stars were removed on the basis of the spectral classification and the catalog of Renson et al. (1991). Binary stars were discarded on the basis of HiPPARcos data (ESA 1997) and the catalog of spectroscopic binaries from Pédoussaut et al. (1985). For A0-A1 stars, these criteria reduced the size of the original sample by about one third.

This paper focuses on a subsample of the A0-A1 normal stars, selected on their low $v \sin i\left(\leq 65 \mathrm{~km} \mathrm{~s}^{-1}\right)$, in order to investigate the slow rotator part of the distribution and accurately check whether these stars are normal or harbor signatures of multiplicity and/or chemical peculiarity. Using this criterion, 73 stars are selected on the whole sky. Among them, 47 can be observed from Observatoire de Haute-Provence (OHP). Table 1 lists the 47 targets defining our sample, together with their spectral type, $V$ magnitude, $v \sin i$ (Royer et al. 2002b) and the different parameters derived in the next sections.

\subsection{Spectroscopic observations}

Spectra of our targets were collected at OHP and observations were spread over an eight year period. The first two runs (April 2005 and June 2006) used the ÉLODIE spectrograph ( $R \approx 42000$, Baranne et al. 1996). Then ÉLODIE was decommissioned in August 2006 and replaced with a more efficient instrument offering a higher spectral resolution: SOPHIE
( $R \approx 75000$, Perruchot et al. 2008). The last part of our program used SOPHIE, in three different runs: July 2009, February 2011 and February 2012. Archival data have also been used: about half the ÉLODIE spectra were taken from the ÉLODIE archive ${ }^{1}$ (Moultaka et al. 2004) and spectra of Vega were taken from the SOPHIE archive $^{2}$. Table 2 lists the different observations of our targets, indicates the corresponding instrument, the observation date, the number of co-added spectra, the modified Julian date at the center of the exposure(s), the signal-to-noise ratio $(\mathrm{S} / \mathrm{N})$ derived using the DER_SNR algorithm (Stoehr et al. 2008) and the measured radial velocity, corrected from the barycentric motion (see Sect. 3). The initial observational strategy was derived from the twofold goal of our program: (i) obtain good S/N spectra $(\approx 150-200)$ to perform, using synthetic spectra at low $v \sin i$, a detailed abundance analysis of unblended weak lines, allowing us to identify the normal stars; (ii) focus on these stars and obtain high $\mathrm{S} / \mathrm{N}$ spectra $(\approx 400)$ to analyze the line profiles and search for gravity-darkening signatures.

\subsection{Data reduction}

For both ÉLODIE and SOPHIE, data are automatically reduced to produce $1 \mathrm{D}$ extracted and wavelength calibrated échelle orders. When stars are observed several times during one observation night, the corresponding spectra are coadded. Then, for each reduced spectrum, échelle orders are normalized separately, using a Chebychev polynomial fit with sigma clipping, rejecting points above $6 \sigma$ or below $1 \sigma$ of the continuum. Normalized orders are merged together, weighted by the blaze function and resampled in a constant wavelength step $\Delta \lambda=0.02 \AA$. Only the spectral intervals outside the wings of Balmer lines and the atmospheric telluric bands are finally retained: 4150-4300 $\AA$,

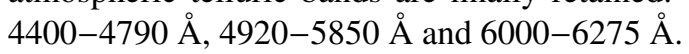

\subsection{Completeness}

Besides selections in $v \sin i$ and the absence of spectroscopic peculiarities, the aforedescribed sample is magnitude-limited and censored in declination and spectral type. These censorships can be applied to the Hipparcos catalog (ESA 1997), complete down to $V=7.3$ (Perryman et al. 1997), to estimate the completeness of the sample. The selection criteria are the following:

- declination $\delta$ higher than $-15^{\circ}$ to reproduce the observability bias due to the location of OHP (i.e. $63 \%$ of the celestial sphere),

- spectral class containing A0 or A1, and luminosity class either $\mathrm{V}, \mathrm{IV} / \mathrm{V}$ or IV, to reproduce the selection made in $\mathrm{RZG}$, - magnitude $V$ brighter than 6.65.

This selection results in 303 stars from the Hipparcos catalog, among them 240 belong to the sample studied by RZG. The star counts per bin of $V$-magnitude are compared in Fig. 1 . The ratio of these two counts gives a completeness of about $80 \%$.

When limited to normal stars, using the results from RZG, 151 stars remain out of the 240 . Our 47 targets correspond to the $v \sin i$-truncated subsample out of these 151 stars.

\footnotetext{
1 http://atlas.obs-hp. fr/elodie

2 http://atlas.obs-hp.fr/sophie
} 


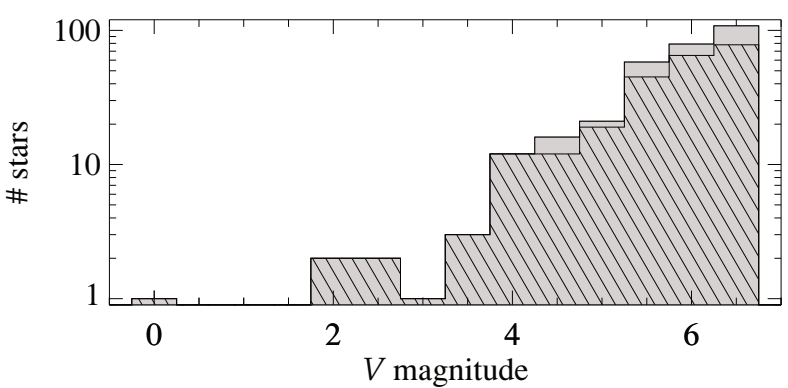

Fig. 1. Counts in $V$ magnitude bins for northern $\left(\delta>-15^{\circ}\right) \mathrm{A} 0-\mathrm{A} 1$ main sequence stars in the HIPPARcos catalog (gray) and in the $v \sin i$ sample (hatched).

\section{Radial velocities}

The normalized spectra are cross-correlated with a synthetic template extracted from the POLLUX database ${ }^{3}$ (Palacios et al. 2010) corresponding to the parameters $T_{\text {eff }}=9500 \mathrm{~K}, \log g=4$ and solar metallicity (computed with sYNSPEC 48, Hubeny \& Lanz 1992), to compute the cross-correlation function (CCF). The radial velocity is derived from the parabolic fit of the upper part (10\%) of the CCF and the values are given in Table 2. The error on the radial velocity is determined from the CCF using the formulation given by Zucker (2003) and assuming the parabolic fit of the CCF.

\subsection{Combining ÉLODIE and SOPHIE}

Fourteen of our targets have spectra collected with both ÉLODIE and SOPHIE. In order to combine the different velocities and detect possible variations, the radial velocity offset between both instruments has to be retrieved. Boisse et al. (2012) derive a relation giving this offset as a function of the $B-V$ color index for late-type stars $(\mathrm{G}$ to $\mathrm{K})$. The offset $\Delta_{\mathrm{E}-\mathrm{S}}$ ranges from 0 to $-0.25 \mathrm{~km} \mathrm{~s}^{-1}$ for these spectral types.

To constrain this offset for the considered spectral type, publicly available spectra of Vega (HD 172167) are retrieved from the ÉLODIE and SOPHIE archives (16 and 10 observations, respectively, see Table 2), and radial velocities are derived the exact same way. The respective average velocities are: $-13.44 \pm 0.05 \mathrm{~km} \mathrm{~s}^{-1}$ and $-13.46 \pm 0.07 \mathrm{~km} \mathrm{~s}^{-1}$. The offset for the considered spectral type is then defined as the difference between these average velocities:

$\Delta_{\mathrm{E}-\mathrm{S}}(\mathrm{A} 0)=0.02 \pm 0.09 \mathrm{~km} \mathrm{~s}^{-1}$.

This offset is not significantly different from zero, and we choose not to correct the derived radial velocities for our targets.

\subsection{Suspected binaries}

The suspicion of binarity from the CCF is raised by the variation of the radial velocity, when several observations are available, and/or by an asymmetric shape of individual CCF.

The variation is taken as significant when the ratio of the external error over the internal error is $E / I \gtrsim 2$ (Abt et al. 1972). The external error is chosen as the standard deviation of the measurements for the different available observations, and the internal error is the one estimated using the formulation from Zucker (2003), which increases with the rotational broadening. Table 3 lists the ten stars with $E / I>2$. The number of observations for

\footnotetext{
3 http://pollux.graal. univ-montp2.fr
}

Table 3. List of targets showing a variation in their radial velocity measurements.

\begin{tabular}{lcccc}
\hline \hline HD & $N$ & $\begin{array}{c}\langle\mathrm{RV}\rangle \\
\left(\mathrm{km} \mathrm{s}^{-1}\right)\end{array}$ & $\begin{array}{c}E \\
\left(\mathrm{~km} \mathrm{~s}^{-1}\right)\end{array}$ & $\begin{array}{c}I \\
\left(\mathrm{~km} \mathrm{~s}^{-1}\right)\end{array}$ \\
\hline 1561 & 2 & -9.2 & 13.01 & 0.64 \\
20149 & 5 & -11.0 & 1.33 & 0.20 \\
46642 & 2 & 35.0 & 4.80 & 0.73 \\
72660 & 4 & 4.2 & 0.70 & 0.03 \\
119537 & 2 & -3.8 & 82.92 & 0.46 \\
156653 & 2 & 3.3 & 1.32 & 0.57 \\
174567 & 2 & -12.5 & 2.36 & 0.46 \\
176984 & 2 & -45.7 & 2.78 & 0.50 \\
196724 & 2 & -20.1 & 2.59 & 0.41 \\
199095 & 2 & -10.8 & 20.14 & 0.49 \\
\hline
\end{tabular}

Notes. Columns: the number of observations $(N)$, the average barycentric radial velocity $\langle\mathrm{RV}\rangle$, the external error $E$ and the internal error $I$.

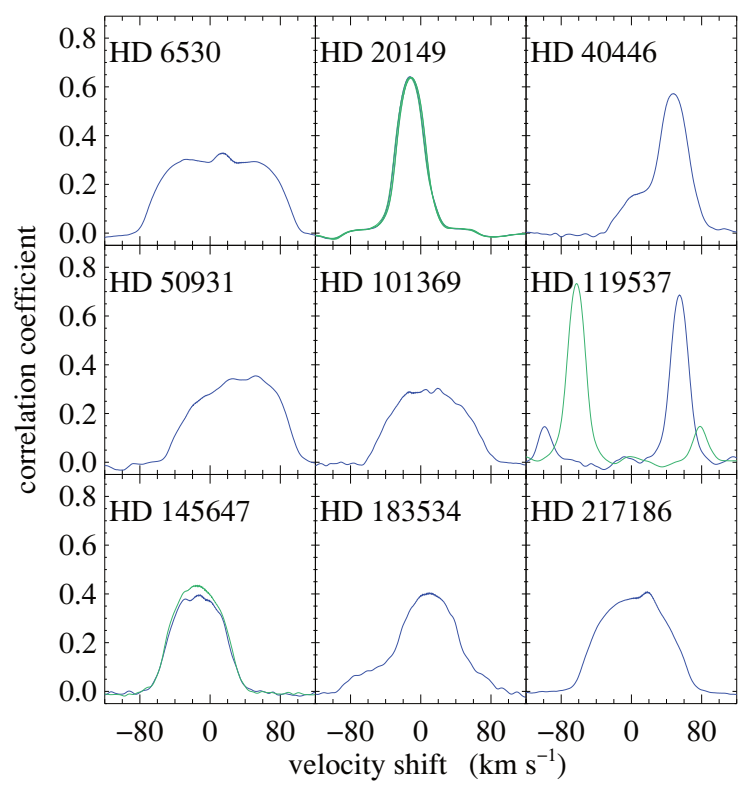

Fig. 2. Cross-correlation functions of suspected binary stars from their asymmetric profiles. When available, several observations are overplotted. The velocity axis takes the barycentric correction into account.

a given star remains small, as a radial velocity follow-up was not intended. These stars are suspected single-lined spectroscopic binaries (SB1).

The stars that display an asymmetric CCF are shown in Fig. 2. For two of them, several observations are available, and a variable radial velocity is noticed. The objects are suspected to be double-lined spectroscopic binaries (SB2).

Details on them and comparison with literature data can be found in Appendix A.

\section{Atmospheric parameters}

We use the revised version of the UVBYBETA code written by Napiwotzki et al. (1993) in order to derive effective temperatures $\left(T_{\text {eff }}\right)$ and surface gravities $(\log g)$. The UvBYBETANEW relies on the calibration of the Strömgren photometry indices $u v b y \beta$ in terms of $T_{\text {eff }}$ and $\log g$. The photometric data are taken from Hauck \& Mermilliod (1998). The derived fundamental parameters are displayed in Table 1. According to Napiwotzki et al. (1993), errors on effective temperature are of the order of $2 \%$ 


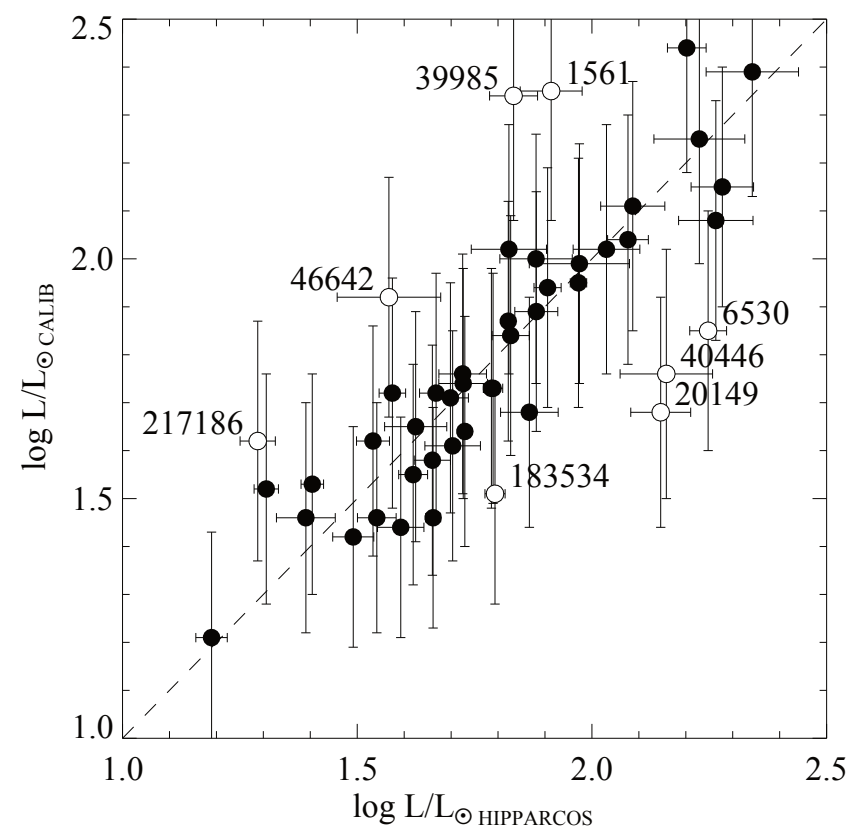

Fig. 3. Consistency check using the luminosity derived from the Torres et al. (2010) calibration and the HipPARcos parallaxes. The dashed line is the one-to-one relation. The outliers are indicated by open symbols, together with their HD number.

for $T_{\text {eff }}<10000 \mathrm{~K}$. The accuracy of surface gravity ranges from $\approx 0.1$ dex for early A-type stars to $\approx 0.25$ dex for hot B stars. In our study we fix the errors on $T_{\text {eff }}$ and $\log g$ to be $\pm 125 \mathrm{~K}$ and \pm 0.2 dex, respectively.

A consistency check on $T_{\text {eff }}$ and $\log g$ is performed by comparing the luminosity derived from the radius calibration (Torres et al. 2010), and the luminosity derived from HIPPARcos parallaxes. Torres et al. (2010) give a polynomial expression of the stellar radius as a function of $T_{\text {eff }}, \log g$ and $[\mathrm{Fe} / \mathrm{H}]$ values. The luminosity is then derived from the Stefan-Boltzmann law. Absolute magnitudes are derived from the Hipparcos parallaxes (van Leeuwen 2007) and from bolometric corrections in the $V$-band, interpolated in the tables from Bessell et al. (1998). The adopted bolometric luminosity parameter $\log L / L_{\odot}$, given in Table 1 , is derived by adopting $M_{\odot}^{\text {bol }}=4.75$ (Allen 1973). Figure 3 compares the luminosity values. The uncertainty from the calibrated luminosity is dominated by the $\log g$ uncertainty. In most cases the two agree to within the uncertainties, but a few outliers are present. These eight stars are indicated in Fig. 3 and seven of them are suspected binaries from the previous section. The new outlier is HD 39985 (see Appendix A). In this plot, HD 33654 is out of range; the low gravity $(\log g=2.9)$ derived from the photometry indicates a giant star, which is confirmed by the luminosity derived from the HipParcos data: $\log L / L_{\odot}=3.63 \pm 0.84$, far brighter than the main-sequence. It is misclassified as a class $\mathrm{V}$ luminosity star.

At this point, the following stars are considered as SB2 with atmospheric parameters contaminated by their multiplicity (no abundances are derived): HD 1561, HD 6530, HD 20149, HD 39985, HD 40446, HD 46642, HD 50931, HD 101369, HD 119537, HD 145647, HD 183534, HD 217186. In addition, the following stars are considered as binaries without contamination of their atmospheric parameters: HD 72660, HD 156653 , HD 174567, HD 176984, HD 196724, HD 199095. HD 33654 is also discarded from the sample as we focus on main-sequence stars.

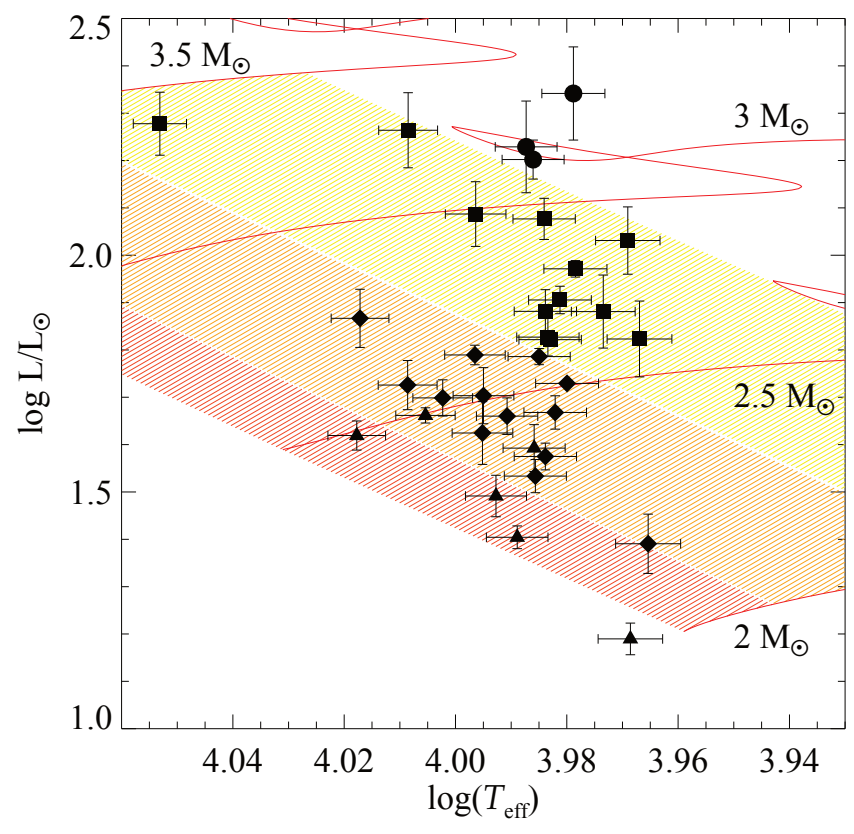

Fig. 4. H-R diagram of the sample. Stars are plotted with different symbols according to their $\log g$, and the same limits are used to define regions from the model gravity: triangles for $4.2 \leq \log g$ (red), diamonds for $3.95 \leq \log g<4.2$ (orange), squares for $3.65 \leq \log g<3.95$ (yellow) and circles for $\log g<3.65$. Evolutionary tracks from BaSTI (Pietrinferni et al. 2006), for $Z=0.0198$, with overshooting, are overplotted for the indicated stellar masses.

Figure 4 shows the non-SB2 stars of the sample in the $\mathrm{H}-\mathrm{R}$ diagram. Luminosities are derived from the trigonometric parallaxes. Evolutionary tracks from BaSTI ${ }^{4}$ (Pietrinferni et al. 2006) are computed with overshooting and for a solar metallicity of $Z=0.0198$. Considering the end of the main-sequence as its reddest point, just before the hook of the evolutionary track when the overall contraction starts, we chose three limits in $\log g$ plotted in Fig. 4. The first two limits in $\log g$ correspond to about one third and two thirds of lifetime on the main sequence for our mass range, according to BaSTI models. At the end of the main sequence the gravity given by BaSTI models is about $\log g=3.5$ for our mass range, and the last limit, $\log g=3.65$, corresponds to about $99 \%$ of the lifetime on the main sequence and defines a region that does not overlap with the hook in terms of gravity and position in the H-R diagram.

These fundamental parameters are used to calculate LTE model atmospheres using ATLAS9 (Kurucz 1993). The ATLAS9 code assumes a plane-parallel geometry, a gas in hydrostatic and radiative equilibrium, and LTE. As explained in Gebran et al. (2008), ATLAS9 model atmospheres are calculated assuming Grevesse \& Sauval (1998) solar abundances and using the prescriptions given by Smalley (2004) for convection.

\section{Rotational velocities}

The $v \sin i$ values used for the selection of this sample are taken from Royer et al. (2002b) who combine determinations from Fourier analysis with the catalog published by Abt \& Morrell (1995). Although these values are statistically corrected from the shift between both scales, this dataset gives the opportunity to derive new and homogeneous projected rotational velocities.

4 http://albione.oa-teramo.inaf.it 


\subsection{Determination of $v \sin i$}

The spectral synthesis described in Sect. 6 provides determinations of $v \sin i$. The same Fourier analysis as in Royer et al. (2002a,b) is also applied to provide an independent determination of $v \sin i$, from the position of the first zero in the Fourier transform (FT) of individual lines.

Díaz et al. (2011) point out the fact that Royer et al. (2002a,b) consider a fixed value of the linear limb-darkening coefficient, $\epsilon=0.6$, to derive $v \sin i$, neglecting the variation of this coefficient with $T_{\text {eff }}, \log g$ and wavelength. The expected variation of the limb-darkening coefficient in our sample stars can be estimated using the values of $\epsilon$ tabulated by Claret (2000). In the Johnson $B$ band, $\epsilon$ varies from 0.64 to 0.56 when the effective temperature increases from 9000 to $11000 \mathrm{~K}$.

In order to improve the determination of $v \sin i, \epsilon$ is taken into account by comparing the position of the first zero with a theoretical rotational profile computed with the linear limbdarkening coefficient derived from the atmospheric parameters of the stars, interpolated in the tabulated values given in Claret (2000), for the $B$ band. The derived value of $\epsilon$ is given in Table 1 .

The line candidates for $v \sin i$ determination are chosen among the list of 23 lines given by Royer et al. (2002b), lying in the spectral range $4215-4577 \AA$. They are retained using criteria based on their shapes in the wavelength domain and in the frequency domain. Error on the $v \sin i$ is taken as the standard deviation of single line determinations. The results are given in Table 1.

\subsection{Rotational velocity scale}

Figure 5a compares the $v \sin i$ derived using FT profiles in the previous paragraph and the ones resulting from the spectral synthesis (described in Sect. 6), from the same data. In this comparison, the suspected SB2 have been discarded. The agreement is very good and the linear relation between both scales is given by:

$v \sin i_{\mathrm{FT}}=(1.08 \pm 0.01) v \sin i_{\mathrm{SYNTH}}-1.8 \pm 0.3$.

The linear fit is overplotted on the data. The slope of the fit shows that above $\sim 30 \mathrm{~km} \mathrm{~s}^{-1}, v \sin i$ derived from FT are slightly higher than the result of the spectral synthesis. This is due to the fact that the FT method uses individual line profiles and is therefore more sensitive to blends than spectral synthesis. As mentioned by Royer et al. (2002b), effects of blends are noticeable on the individual $v \sin i$ when compared with the value derived from Mg II triplet at $4481 \AA$ A. Two stars in Fig. 5a (and Table 1) show significant differences in $v \sin i$ : HD 47863 and HD 223855.

Figure $5 \mathrm{~b}$ compares the new determinations to the homogenized values given by Royer et al. (2002b, RGBGZ). These latter determinations result from the merging of data from $\mathrm{Abt}$ \& Morrell (1995, AM) and values derived from FT. The different symbols in the plot correspond to the combined source: AM only, FT only, or combination of both. The systematic overestimation at low $v \sin i$ in the determination from Royer et al. (2002b) results from the lower spectral resolution of ÉLODIE compared to SOPHIE in this work. A significant shift is noticed with the scaled values from AM, whereas no systematic effect is observed when comparing to values derived from FT. The linear relation between both scales (displayed in Fig. 5b) is given by:

$v \sin i_{\mathrm{FT}}=(1.09 \pm 0.03) v \sin i_{\mathrm{RGBGZ}}+2.8 \pm 1.2$.

The scaling relation between AM and the FT results is derived by Royer et al. (2002b) using spectral types from B8 to F2.
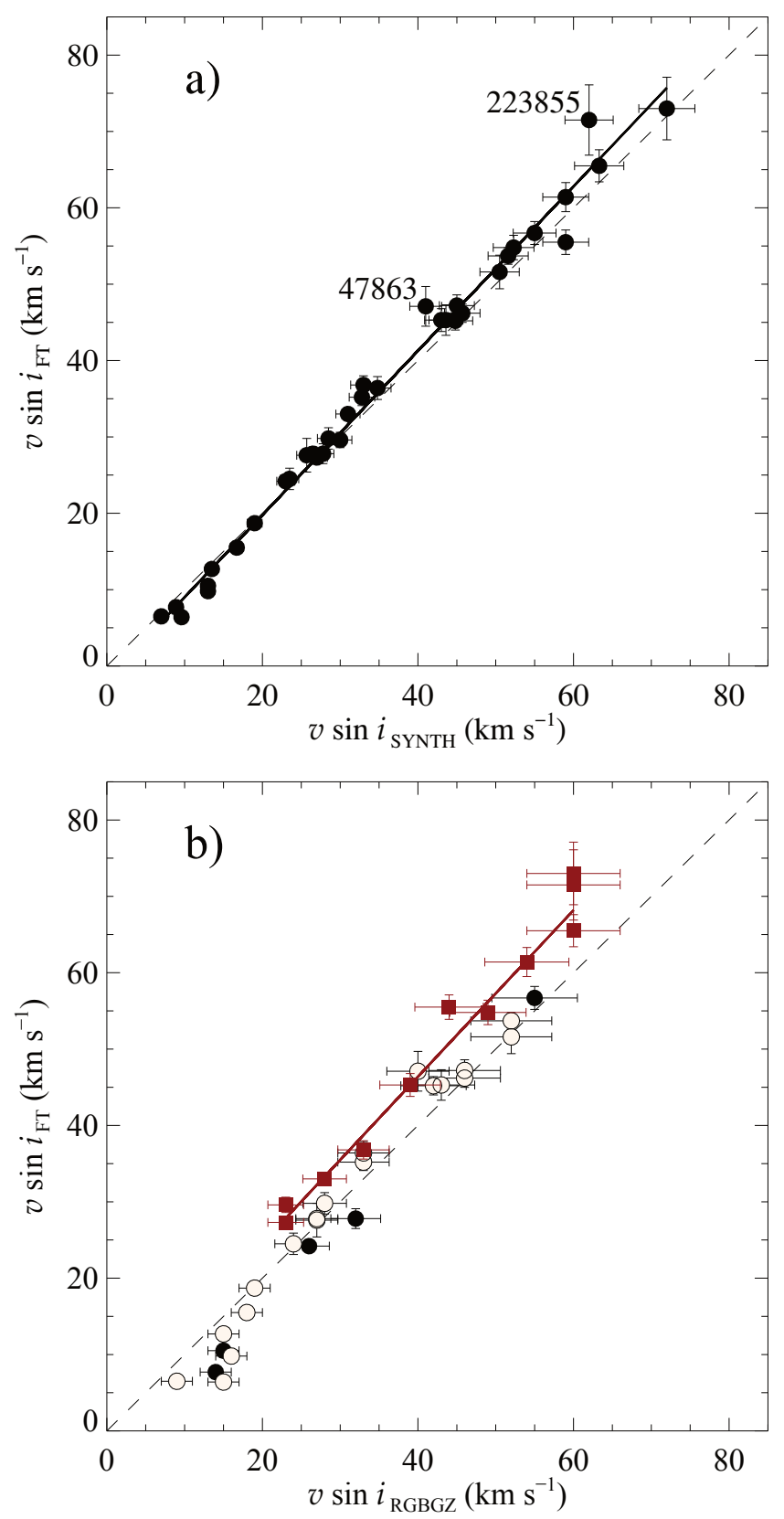

Fig. 5. Comparison of the different $v \sin i$ determinations given in Table 1, excluding SB2 stars. a) The $v \sin i$ derived from the first zero of the FT is compared to the one derived from the spectral synthesis (SYNTH). The linear fit is shown by the thick solid line. The two stars with the largest differences are labeled. b) The $v \sin i$ derived from the first zero of the FT is compared to the homogenized merged catalog from Royer et al. (2002b, RGBGZ). The different symbols stand from the source of the data in the latter: filled squares were from $\mathrm{Abt}$ \& Morrell (1995, AM), filled circles were derived by RGBGZ using Fourier analysis, open circles are a combination of both sources. The linear fit on the subsample with data from AM is represented by the thick solid line. In both panels, the one-to-one relation is represented by the dashed line.

This relation could slightly vary with the spectral type, as the comparison restricted to A0-A1-type stars suggests. The statistical correction applied by Royer et al. (2002b) is $v \sin i=$ $1.05 v \sin i_{\mathrm{AM}}+7.5$ and the resulting values for A0-A1 stars may be undercorrected. 


\section{Abundance analysis}

For the rotational velocity range in our sample $(v \sin i \leq$ $65 \mathrm{~km} \mathrm{~s}^{-1}$ ), the most appropriate method to derive individual chemical abundances is the use of spectrum synthesis technique. Specifically, we iteratively adjust LTE synthetic spectra to the observed ones by minimizing the $\chi^{2}$ of the models to the observations using Takeda's (1995) iterative procedure (see Gebran et al. 2008, for a detailed discussion).

\subsection{Spectrum synthesis}

Takeda's procedure is divided in two parts. The first part is a modified version of Kurucz (1992) WIDTH9 code and computes the opacity data. The second part of the routine computes the synthetic spectrum and minimizes the dispersion between the normalized synthetic spectrum and the observed one.

The line list used for spectral synthesis is the one used in Gebran et al. (2008, 2010). All transitions between 3000 and $7000 \AA$ from Kurucz's gfall.dat ${ }^{5}$ line list are selected for the calculation of the synthetic spectra. The abundance determination relies mainly on unblended transitions for about 14 chemical elements (C, O, Mg, Si, Ca, Sc, Ti, Cr, Fe, Ni, Sr, Y, Zr, and Ba). Most of these lines are weak because they are formed deep in the atmosphere. They are well suited to abundance determinations as LTE should prevail in the deeper layers of the atmospheres.

The accuracy of the atomic parameters (wavelengths, lower excitation potential, oscillator strength and damping constants) is checked against more accurate and/or more recent laboratory determinations, using the $\operatorname{VALD}^{6}$ (Kupka et al. 1999) and the $\mathrm{NIST}^{7}$ databases. The adopted atomic data for each elements are collected in Table B.1, where for each element, the wavelength, the oscillator strength, and the reference are given.

A byproduct of this procedure is the derivation of the rotational $(v \sin i)$ and the microturbulent $\left(\xi_{\mathrm{t}}\right)$ velocities. We first derive the rotational and microturbulent velocities using several weak and moderately strong unblended Fe II lines located between $4491.405 \AA$ and $4522.634 \AA$ and the $\mathrm{Mg}$ II triplet around $4481 \AA$ by allowing small variations around solar abundances of $\mathrm{Mg}$ and $\mathrm{Fe}$ as explained in Sect. 3.2.1 of Gebran et al. (2008). The weak iron lines are very sensitive to rotational velocity but not to microturbulent velocity while the moderately strong Fe II lines are affected mostly by changes of microturbulent velocity. The Mg II triplet is sensitive to both $\xi_{\mathrm{t}}$ and $v \sin i$. The derived rotational and microturbulent velocities are displayed in Table 1. The error on $\xi_{\mathrm{t}}$ is $\pm 0.5 \mathrm{~km} \mathrm{~s}^{-1}$ (Gebran et al. 2010). By testing the effect of the variation of the $v \sin i$ on the abundance derived from the $\mathrm{Mg}$ II triplet, we are able to determine $\Delta(v \sin i)$ that causes a variation of the abundance of about the error level $\left(\Delta[\mathrm{Mg} / \mathrm{H}] \sim \sigma_{\mathrm{Mg}}\right)$. On average we find that the rotational velocities have a precision estimated as $5 \%$ of the nominal $v \sin i$ and are in good agreement with those derived using the Fourier transforms (Sect. 5).

Once the rotational and microturbulent velocities are fixed, we then derive the abundance that minimized the $\chi^{2}$ for each transition of a given chemical element. Figure 6 displays the observed spectra of six A stars, for different $v \sin i$, with their respective best fit synthetic spectra.

\footnotetext{
5 http://kurucz .harvard.edu/LINELISTS/GFALL

6 http://www . astro.uu.se/ vald/php/vald.php

7 http://physics.nist.gov/PhysRefData/ASD/lines_form. html
}

\subsection{Resulting abundances}

The derived abundances are mean values and given in solar scale in Table 4. For a given chemical element $\mathrm{X}$, the abundance $[\mathrm{X} / \mathrm{H}]$ is equal to the difference between the absolute abundance in the star $\log (\mathrm{X} / \mathrm{H})_{\star}$ and the abundance in the Sun $\log (\mathrm{X} / \mathrm{H})_{\odot}$ derived from Grevesse \& Sauval (1998). As done in Gebran et al. (2008), errors on the elemental abundances are estimated by the standard deviation, assuming a Gaussian distribution of the abundances derived from each line. When only one line is measured, we adopted an average error on abundances derived from the results of Gebran et al. $(2008,2010)$ and Gebran \& Monier (2008) for stars with $v \sin i<70 \mathrm{~km} \mathrm{~s}^{-1}$. This error is found to be $\approx 0.15$ dex.

\section{Cluster analysis and classification}

A quick look on the abundances listed in Table 4 and how these data are distributed in the $\mathrm{Sr}-\mathrm{Sc}$ plane (Fig. $7 \mathrm{~b}$ ) suggests that several stars present $\mathrm{CP}$ characteristics. The size of our sample allows the use of statistical tools to disentangle the $\mathrm{CP}$ and normal stars and perform this classification in an automatic way, from the full abundance data set.

\subsection{Classification criteria}

In our temperature domain, the expected CP stars are the metallic Am stars (CP1) and the magnetic Ap stars (CP2). The classical definition of CP1 stars relies on $\mathrm{Ca}, \mathrm{Sc}$, iron-peak elements and heavy elements (Conti 1970; Preston 1974), and for the CP2 stars it is based on $\mathrm{Si}, \mathrm{Cr}, \mathrm{Sr}$ and $\mathrm{Eu}$ (Preston 1974). Among the 14 species studied in this work, 10 species correspond to these classical definitions, i.e $\mathrm{Si}, \mathrm{Ca}, \mathrm{Sc}, \mathrm{Cr}, \mathrm{Fe}, \mathrm{Ni}, \mathrm{Sr}, \mathrm{Y}, \mathrm{Zr}$ and $\mathrm{Ba}$.

\subsection{Hierarchical cluster analysis}

Hierarchical cluster analysis, applied to chemical abundances by Cowley \& Bord (2004), is a bottom-up classification which consists in grouping data by proximity in a given space, producing a classification tree from single elements to the entire sample. It identifies clusters as a function of distance between elements. This method is applied to our data and clusters are searched for in the multidimensional space defined by the abundances from Table 4. Abundances are normalized so that the variation of a given elemental abundance over the full sample lies in the interval $[0,1]$, ensuring that the different elements are equally weighted. Proximity is based on the Euclidean distance in the multidimensional normalized space. In the resulting classification tree (Fig. 7a), two main groups appear. They are identified as $\mathrm{CP}$ and normal stars, based on the median $[\mathrm{Sr} / \mathrm{H}]$ abundance: this element being very discriminant (Fig. 7b), the group showing the highest median $[\mathrm{Sr} / \mathrm{H}]$ abundance is associated with $\mathrm{CP}$ stars, and the other one with normal stars.

The construction of the classification tree does not take errors into account. In order to test the effect of the errors on the resulting memberships, new abundances are randomly simulated by adding Gaussian noise to the measured abundances, using the related standard deviation. Over 5000 simulations, targets are allocated to one group or the other, and the proportion of allocation to one group is taken as the final membership probability. This defines a new classification, taking the abundance errors into account. 


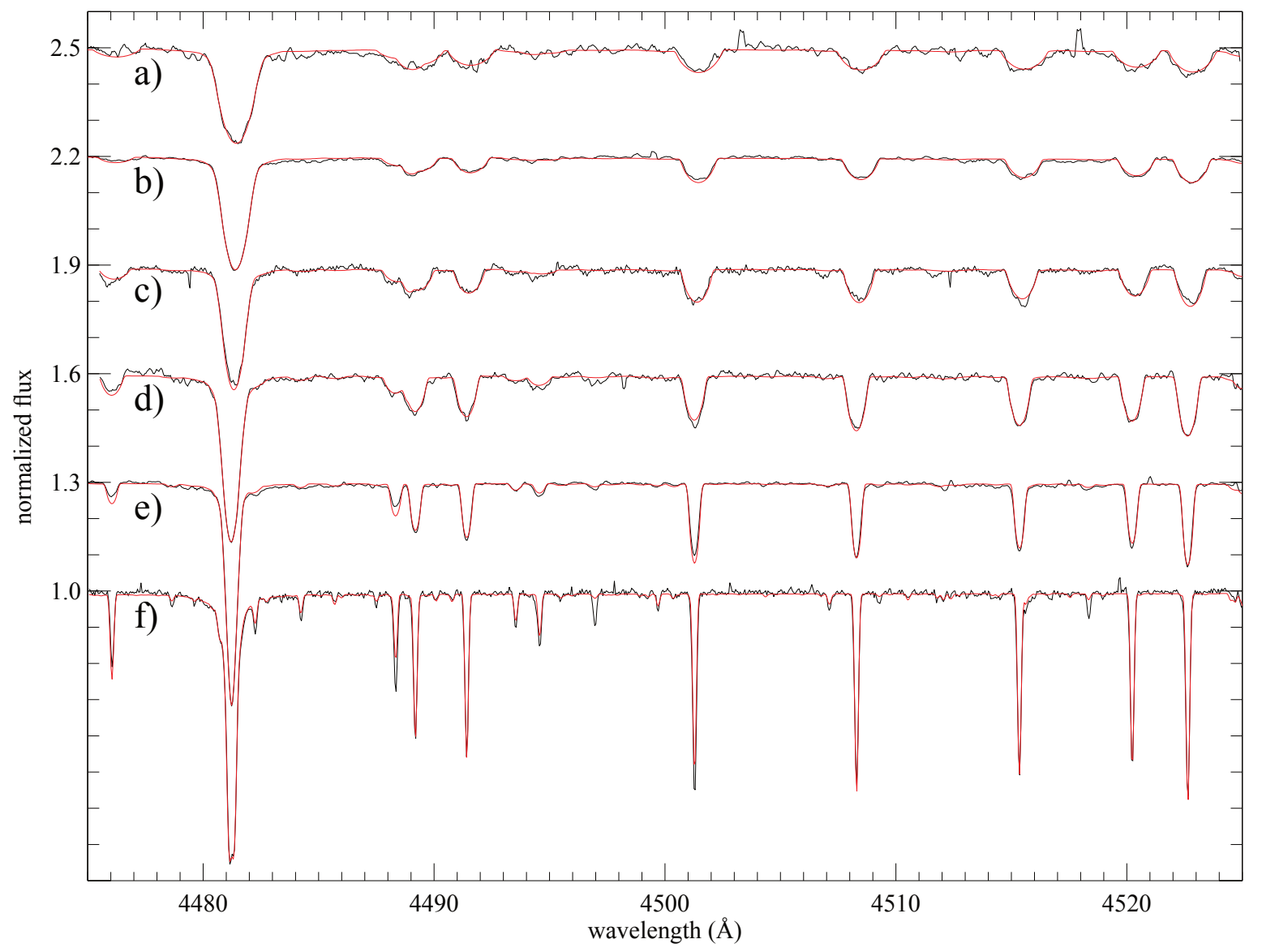

Fig. 6. Selected observed spectra for six A-type stars with different values of $v \sin i$ (in black) shifted in flux by steps of 0.3 for clarity reasons. In red lines are the respective synthetic spectra in a region rich in $\mathrm{Fe}_{\text {II }}$, Ti II, and $\mathrm{Mg}_{\text {II }}$ lines. The spectra are sorted from top to bottom by decreasing $v \sin i$. The stars are a) HD 101369, b) HD 133962, c) HD 107655, d) HD 65900, e) HD 58142 and f) HD 72660. Spectra are shifted to rest wavelengths.

This multivariate statistical analysis is performed using $\mathrm{R}^{8}$ (R Development Core Team 2011).

\subsection{Resulting classification and comparison with literature data}

Hierarchical cluster analysis is applied to the 14 species and produces the membership flag $f_{14}$ ( 1 for $\mathrm{CP}, 0$ for normal) from the direct classification, for each star. The simulations taking errors into account give the membership probability $p_{14}$. Respectively, $f_{10}$ and $p_{10}$ are produced when applying hierarchical cluster analysis to the 10 "classical" species. Figure 8 displays these four criteria, also listed in Table 5.

The four criteria give rather consistent results. The distribution of $p_{14}$ shows slightly more separated and peaked groups than $p_{10}$, as a result of the additional discriminant species. A few objects show discrepant classifications: HD 219485 is classified as probably normal in Table 5 because the classification based on $p_{10}$ points it as a CP star whereas the other criteria indicate it is normal; two objects are classified as uncertain (HD 1439 and HD 219290) because the classification based on 10 species and the one based on 14 species produce contradictory results.

\footnotetext{
$8 \mathrm{R}$ is a language and environment for statistical computing and graphics, available at http://www.r-project.org
}

In order to check how separated are the two groups in the chemical abundance space, we perform a principal component analysis to display the data in fewer dimensions. Principal component analysis is a statistical method that expresses a set of variables, possibly correlated, as a set of linearly uncorrelated variables called principal components. The principal components are linear combinations of the original variables and are defined in such a way that they have the largest possible variance. We apply this method to our data and derive the first two principal components from the 14-species abundances. The determined elemental abundances are projected onto these two new axes in Fig. 9, as well as the 14 axes corresponding to the different species. The first principal component (PC1) derived from the data explains $38.8 \%$ of the total variation and the second one (PC2) explains $15.4 \%$ of the variance in the 14-species abundances. Figure 9 shows that species from titanium to zirconium have strong positive loadings on PC1, whereas oxygen and carbon have strong negative loadings. On the other hand, PC2 has strong loadings from magnesium and silicium. The original data for the 34 stars are expressed in the first two components and overplotted in Fig. 9. They are represented according to the classification resulting from the cluster analysis. The dichotomy is very clear along the first principal component, and the objects identified as "uncertain" in Table 5 lie between the two groups.

Back in 2007, RZG used the release of the General Catalog of Ap and Am stars from Renson et al. (1991) to identify 

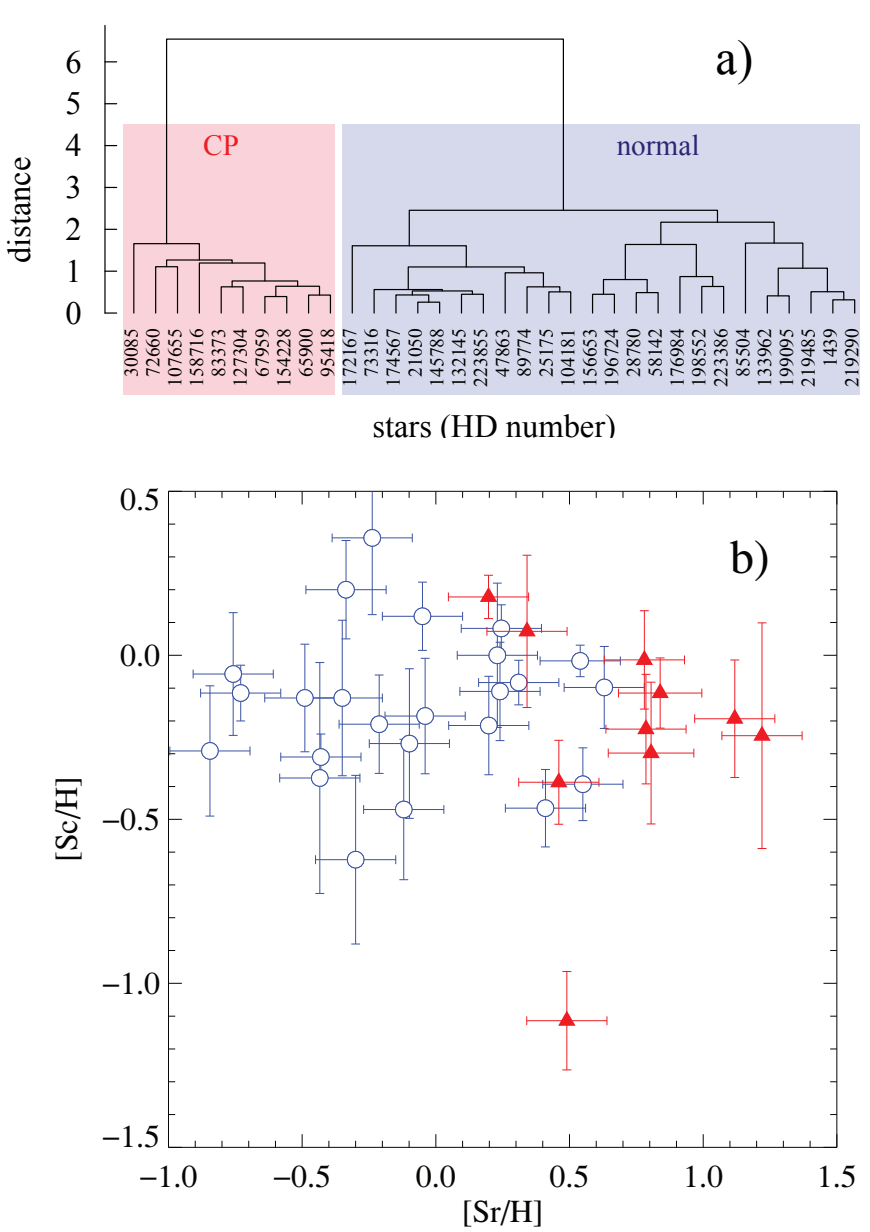

Fig. 7. a) Dendogram plot of the hierarchical tree resulting from the cluster analysis of the 14-species chemical abundances of the sample without errors. The $x$-axis gives the HD numbers of the stars and the $y$-axis represents the Euclidean distance, in the normalized abundance space, between subgroups. The two main groups are identified by the labeled boxes. b) Scandium abundances as a function of strontium. The two main groups identified above are projected with different symbol: filled triangle stand for CP stars, open circles represent normal stars.

the chemically peculiar stars. This release is now outdated by Renson \& Manfroid (2009). The 47 targets of our sample are not present in the previous version of the catalog, and the classification results can be compared with the content of the new release. Among these objects, nine are present in the catalog from Renson \& Manfroid (2009), and are listed in Table 6. For two thirds of the common stars, both classifications are in agreement.

On the other hand, four stars are classified as peculiar but are absent from the catalog built by Renson \& Manfroid (2009): HD 30085, HD 65900, HD 67959, HD 158716. More details can be found in Appendix A.

\section{Discussion}

\subsection{Abundance patterns}

Figure 10 displays the median abundance patterns for the normal and peculiar stars resulting from our classification. Only fully agreeing classifications (all four criteria) are used to derive the median abundances, i.e. 21 normal stars and $10 \mathrm{CP}$

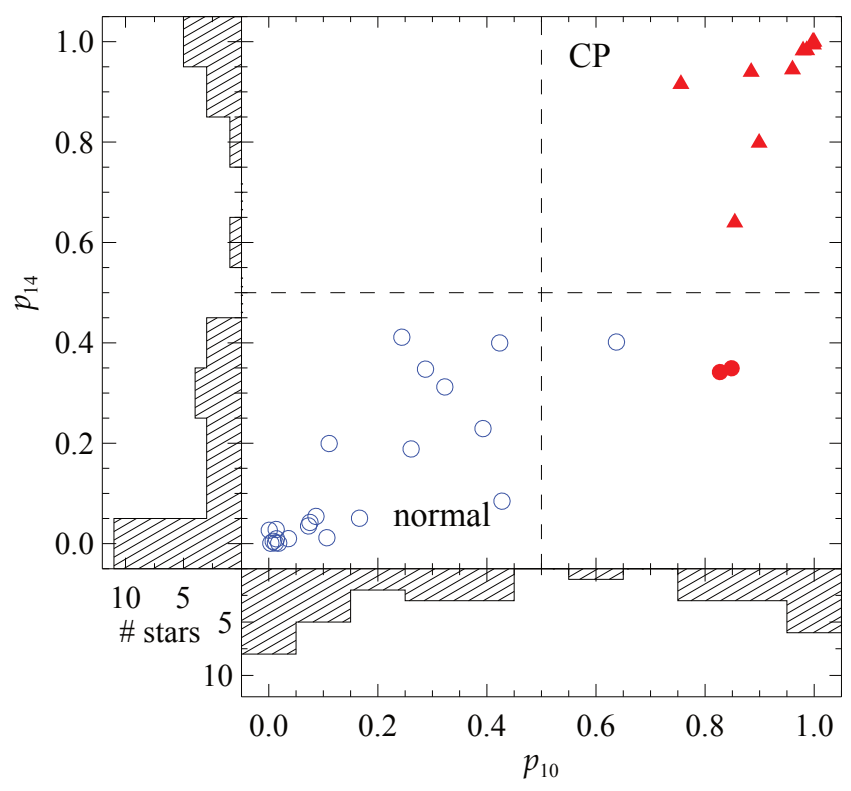

Fig. 8. Membership to the $\mathrm{CP}$ group from the four criteria: probabilities using 10 and 14 species ( $p_{10}$ and $p_{14}$, respectively), direct classifications using 10 species (filled symbol for CP stars, open symbol for normal stars), and using 14 species (triangle for CP stars, circle for normal stars). Lower and left panels are the projected distributions of $p_{10}$ and $p_{14}$, respectively.

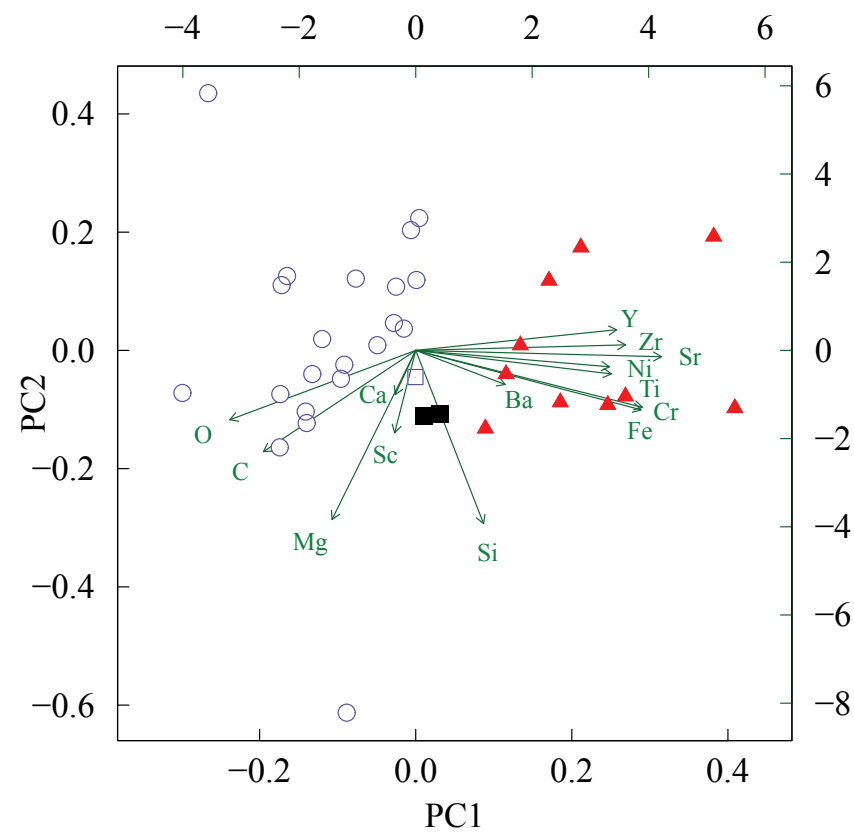

Fig. 9. Observed sample and species projected on the first and second principal components (see text). The CP and normal stars are indicated by filled triangles and open circles, respectively, according to the results from the cluster analysis. Filled squares represent the two uncertain objects and the open square stands for the probable normal star. The directions of the arrows show the relative loadings of the species on the first and second principal components.

stars. The dispersions are represented by the 16th and 84th percentiles, which in the Gaussian case correspond to the $\pm 1 \sigma$ standard deviation.

The abundance pattern for the normal stars is compared with data from the Pleiades (Gebran \& Monier 2008) and 

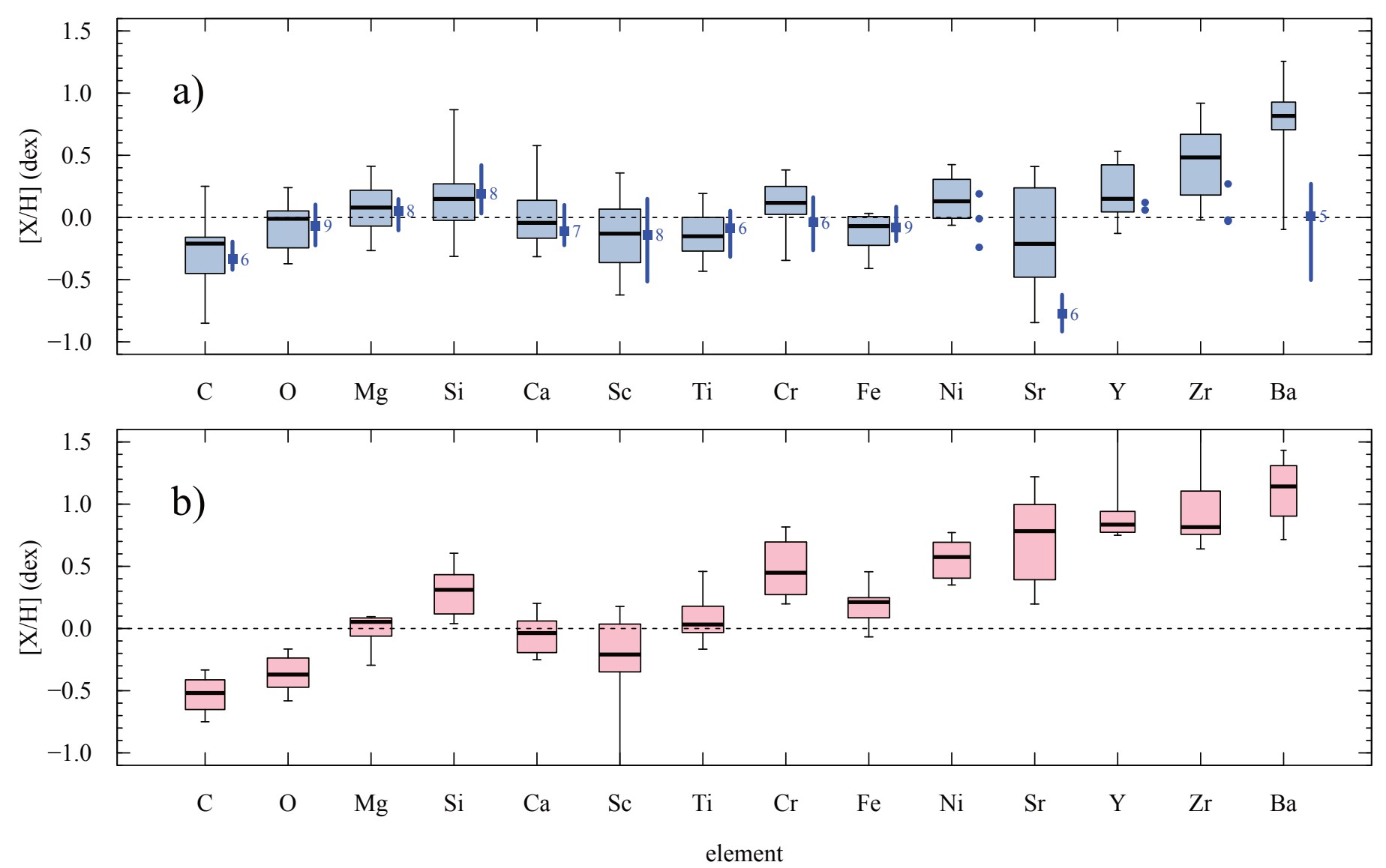

Fig. 10. Abundance patterns are displayed as box and whisker plots for a) the normal stars and b) the CP stars. Boxes correspond to the 16th and 84th percentiles of the abundance distribution for each element, thick horizontal lines represent the median value, and the whiskers span from the minimum to the maximum values. The width of the boxes is proportional to the square root of the number of values. The horizontal dashed line stands for the solar values. In the top panel, median values and 16th and 84th percentiles derived from the comparison stars are plotted on the right side of the boxes, as filled squares and thick vertical lines. The number of abundance determinations is indicated when five or more abundances are available, and all individual determinations are displayed when the number is less than five (see Sect. 8.1).

Table 6. Comparison of the classification with the General Catalog of Ap and Am stars (Renson \& Manfroid 2009).

\begin{tabular}{llcc}
\hline \hline HD & \multicolumn{2}{c}{ Renson \& Manfroid (2009) } & This work \\
\hline 58142 & & A0-A2 & normal \\
72660 & & A1- & CP \\
83373 & $?$ & A1 Si & CP \\
85504 & & A1 Mn & normal \\
95418 & & A0- Ba Y & CP \\
107655 & $?$ & A1- & CP \\
127304 & $?$ & B9 Si & CP \\
145788 & $?$ & A1 Si & normal \\
154228 & $?$ & A1 Si & CP \\
\hline
\end{tabular}

Notes. In the classification from Renson \& Manfroid (2009), a dash indicates an Am star and no dash indicates an Ap star. The question mark indicates doubtful cases.

from the Ursa Major moving group (Monier 2005). We select nine normal A-type stars corresponding to our observed effective temperature range $\left(T_{\text {eff }}>8900 \mathrm{~K}\right)$ : six stars are members of the Pleiades (HD 23763, HD 23948, HD 23629, HD 23632, HD 23489, HD 23387) and three belong to Ursa Major (HD 1404, HD 12471, HD 209515). They are analyzed by the latter authors using the same method and the code from Takeda (1995), ensuring a comparison with homogeneous data. Their median abundance pattern and the corresponding percentiles are overplotted in Fig. 10a. It should be noted that this comparison sample is small and does not fully cover all the species. The number of available determinations for each species in the comparison sample is indicated in the plot. The agreement between both patterns is very good but we notice discrepancies for strontium and the heavier elements. The comparison stars have on average a larger rotational broadening: seven of them have $100 \leq v \sin i \leq 200 \mathrm{~km} \mathrm{~s}^{-1}$.

As far as the abundance pattern for the CP stars is concerned, no object shows a significant underabundance in calcium and the $\mathrm{Ca}$ abundance is very similar between $\mathrm{CP}$ and normal stars. This tendency is possibly due to the fact that previously known $\mathrm{CP}$ stars are not part of our sample. We find that carbon and oxygen are underabundant, in agreement with Roby \& Lambert (1990). The iron-peak elements and heavy elements are overabundant, compared to the normal stars.

By construction, the $v \sin i$ range is limited in our sample. No clear trend of abundances versus $v \sin i$ is detected in our data, neither on the whole dataset nor considering both groups separately.

\subsection{Rotational velocity distribution}

Sections 3, 4 and 7 show that our sample is still contaminated by $\mathrm{CP}$ stars and binary stars. These new identifications allow 


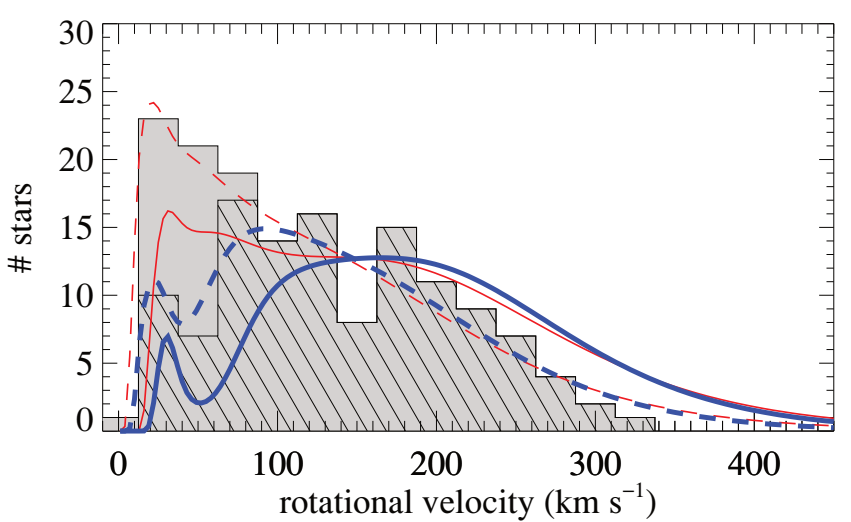

Fig. 11. Distributions of rotational velocities: histograms are the observed $v \sin i$ (hatched histogram corresponds to the cleaned sample); dashed lines stand for the smoothed distribution of projected rotational velocities; solid lines are the distributions of equatorial velocities. Thick lines correspond to the newly cleaned sample of 121 stars whereas thin lines represent the full sample of 151 stars.

the analysis of the rotational velocity distribution of normal stars with a much cleaner sample. It can be noticed that in our sample, no CP stars were found with $v \sin i \gtrsim 45 \mathrm{~km} \mathrm{~s}^{-1}$. The contamination by binary stars remains larger than by $\mathrm{CP}$ stars.

As defined in Sect.2.4, our sample of 47 stars is the low $v \sin i$ part of a larger sample of 151 normal A0-A1 stars representing an $80 \%$-complete, magnitude-limited volume. The distribution of rotational velocities of normal A0-A1 can be analyzed with this larger sample. The gray histogram in Fig. 11 represents the distribution of their observed $v \sin i$. By removing the 30 stars identified as peculiar and/or binaries in the previous sections, a cleaned subsample of 121 stars is selected, and its distribution of $v \sin i$ is showed by the hatched histogram (Fig. 11). The smoothed distributions of $v \sin i$ is obtained by applying the method described in Bowman \& Azzalini (1997) and ported to R (R Development Core Team 2011), and are shown by the dashed lines in Fig. 11. These distributions are then rectified from the projection effect to recover the distributions of equatorial velocities, assuming the rotation axes are randomly oriented (see RZG for details).

The high probability density for slow velocities disappears from the distribution in the cleaned sample, but a significant proportion (about 14\%) of the normal stars rotates slowly at $v \leq 100 \mathrm{~km} \mathrm{~s}^{-1}$. These results considerably reduce the presence of slowly rotating normal A0-A1 stars. The overdensity at $v \leq 50 \mathrm{~km} \mathrm{~s}^{-1}$ represents $4 \%$ of the distribution, i.e. five stars.

In the sample, the normal star with the lowest $v \sin i$ is HD $145788\left(v \sin i=9.8 \mathrm{~km} \mathrm{~s}^{-1}\right.$ as measured from the ÉLODIE spectrum). In the rectified distribution, there is no star rotating more slowly than $v=20 \mathrm{~km} \mathrm{~s}^{-1}$.

\section{Summary and conclusion}

This work provides the spectroscopic study of a sample of 47 A0-A1 stars, initially selected from Royer et al. (2002b, 2007 ) to be main-sequence, low $v \sin i$, normal stars. The analysis of the cross-correlation profiles, and the variation of radial velocities allow the identification of suspected spectroscopic binaries. The spectral synthesis and the determination of chemical abundances are used to identify chemically peculiar stars using a hierarchical classification. The results reveal that two thirds of the sample is composed of spectroscopic binaries and chemically

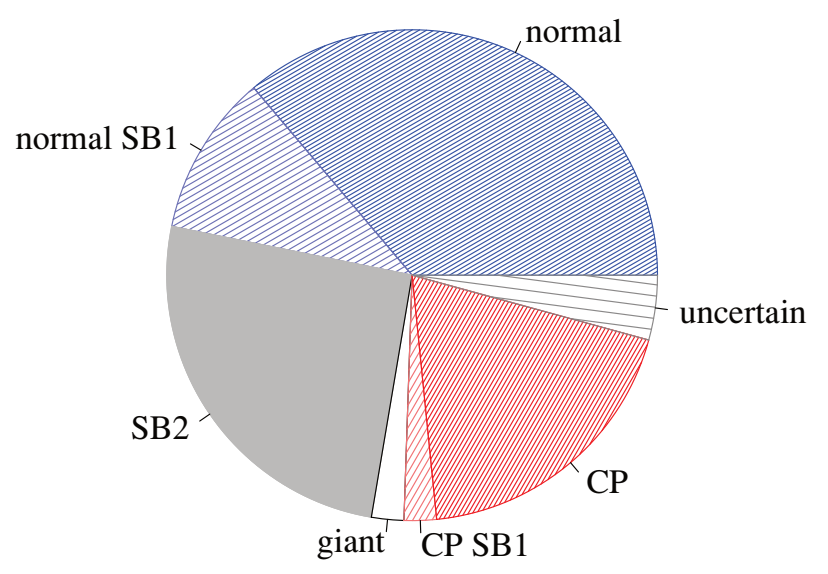

Fig. 12. Resulting content of the sample of $47 \mathrm{~A} 0-\mathrm{A} 1$ stars.

Table 7. List of HD identifiers for the single normal stars.

\begin{tabular}{lcccccc}
\hline \hline 21050 & 25175 & $\mathbf{2 8 7 8 0}$ & 47863 & $\mathbf{5 8 1 4 2}$ & 73316 & 85504 \\
89774 & 104181 & 132145 & 133962 & 145788 & 172167 & 198552 \\
$\mathbf{2 1 9 4 8 5}$ & 223386 & 223855 & & & & \\
\hline
\end{tabular}

Notes. The ones written in boldface are suspected to be doubtful candidates from the comparison with literature data (see Appendix A).

peculiar stars, and only 17 stars turn out to be normal, showing no sign of multiplicity nor peculiarity. The final composition of the sample is given by the pie chart in Fig. 12 .

In the framework of a nearly complete, magnitude limited sample of 121 A0-A1-type normal stars, this implies that the distribution of equatorial velocities, under the assumption of randomly oriented rotation axes, is no longer dominated by a large fraction of slowly rotating stars. Only $14 \%$ of the A0-A 1 normal stars are rotating at $v \leq 100 \mathrm{~km} \mathrm{~s}^{-1}$, which correspond to about 16 objects, and $21 \%$ are rotating at $v \leq 120 \mathrm{~km} \mathrm{~s}^{-1}$. This is to be compared with the corresponding proportions in the noncleaned sample: $31 \%$ and $37 \%$, respectively (Fig. 11). The distribution is not bimodal as previously claimed by RZG, but the overlap with the distribution of equatorial velocity of CP stars seems real, contrary to the conclusion of Abt \& Morrell (1995) and Abt (2000).

The 17 normal stars, spectroscopically identified in this paper, are listed in Table 7. The objects were not extensively monitored in terms of radial velocity and spectroscopic binarity may still remain undetected in this sample. Possibly doubtful candidates are indicated in Table 7, which could be either spectroscopic binaries or marginal CP stars (see Appendix A). The 17 normal stars will be more deeply investigated in a forthcoming paper to check whether signatures of gravity darkening due to fast rotation seen pole-on are present in the spectra. If rotation axes are randomly oriented, the probability to observe a star with $i \leq 10^{\circ}$ is about $1.5 \%$, which would produce just two stars among the 121 normal A0-A1 stars.

Acknowledgements. We are very thankful to the referee for his/her appropriate and constructive suggestions and his/her proposed corrections to improve the paper. This work has made use of BaSTI web tools (v. 5.0.1). This research has made use of the VizieR catalog access tool, CDS, Strasbourg, France. We are grateful to S. Ilovaisky for providing calibration data corresponding to SOPHIE observations of Vega. 
F. Royer et al.: Normal A0-A1 stars with low rotational velocities. I.

\section{References}

Abt, H. A. 2000, ApJ, 544, 933

Abt, H. A. 2009, AJ, 138, 28

Abt, H. A., \& Morrell, N. I. 1995, ApJS, 99, 135 (AM)

Abt, H. A., \& Moyd, K. I. 1973, ApJ, 182, 809

Abt, H. A., Levy, S. G., \& Gandett, L. 1972, AJ, 77, 138

Adelman, S. J. 1994, MNRAS, 271, 355

Adelman, S. J. 2004, in The A-Star Puzzle, eds. J. Zverko, J. Žižňovský, S. J. Adelman, \& W. W. Weiss, IAU Symp. 224, 1

Adelman, S. J., \& Pintado, O. I. 1997, A\&A, 125, 219

Adelman, S. J., Yu, K., \& Gulliver, A. F. 2011, Astron. Nachr., 332, 153

Allen, C. W. 1973, Astrophysical quantities, 3rd edn. (The Athone Press University of London)

Altmann, M., \& de Boer, K. S. 2000, A\&A, 353, 135

Aristidi, E., Carbillet, M., Prieur, J.-L., et al. 1997, A\&AS, 126, 555

Baranne, A., Queloz, D., Mayor, M., et al. 1996, A\&AS, 119, 373

Bessell, M. S., Castelli, F., \& Plez, B. 1998, A\&A, 333, 231

Biemont, E., Grevesse, N., Hannaford, P., \& Lowe, R. M. 1981, ApJ, 248, 867

Biermann, L., \& Lübeck, K. 1948, Z. Astrophys., 25, 325

Boisse, I., Pepe, F., Perrier, C., et al. 2012, A\&A, 545, A55

Bowman, A. W., \& Azzalini, A. 1997, Applied smoothing techniques for data analysis: the kernel approach with S-plus illustrations, Oxford statistical science series No. 18 (Oxford: Clarendon Press)

Claret, A. 2000, A\&A, 363, 1081

Conti, P. S. 1970, PASP, 82, 781

Cowley, C. R., \& Bord, D. J. 2004, in The A-Star Puzzle, eds. J. Zverko,

J. Žižňovský, S. J. Adelman, \& W. W. Weiss, IAU Symp., 224, 265

Díaz, C. G., González, J. F., Levato, H., \& Grosso, M. 2011, A\&A, 531, A143

Dworetsky, M. M. 1974, ApJS, 28, 101

ESA 1997, The Hipparcos and Tycho Catalogues, ESA-SP, 1200

Fossati, L., Ryabchikova, T., Bagnulo, S., et al. 2009, A\&A, 503, 945

Fuhr, J. R., Martin, G. A., \& Wiese, W. L. 1988, J. Phys. Chem. Ref. Data, 17

Gebran, M., \& Monier, R. 2008, A\&A, 483, 567

Gebran, M., Monier, R., \& Richard, O. 2008, A\&A, 479, 189

Gebran, M., Vick, M., Monier, R., \& Fossati, L. 2010, A\&A, 523, A71

Gontcharov, G. A. 2006, Astron. Lett., 32, 759

Grenier, S., Burnage, R., Faraggiana, R., et al. 1999, A\&AS, 135, 503

Grevesse, N., \& Sauval, A. J. 1998, Space Sci. Rev., 85, 161

Gulliver, A. F., Hill, G., \& Adelman, S. J. 1994, ApJ, 429, L81

Hauck, B., \& Mermilliod, M. 1998, A\&AS, 129, 431

Hill, G. M. 1995, A\&A, 294, 536

Hill, G., Gulliver, A. F., \& Adelman, S. J. 2010, ApJ, 712, 250

Hubeny, I., \& Lanz, T. 1992, A\&A, 262, 501

Kukarkin, B. V., Kholopov, P. N., Artiukhina, N. M., et al. 1981, Catalogue of suspected variable stars, Moscow, Acad. of Sciences USSR Shternberg, Nachrichtenblatt der Vereinigung der Sternfreunde

Kupka, F., Piskunov, N., Ryabchikova, T. A., Stempels, H. C., \& Weiss, W. W. 1999, A\&AS, 138, 119

Kurucz, R. L. 1992, Rev. Mex. Astron. Astrofis., 23, 45
Kurucz, R. L. 1993, in Space Stations and Space Platforms - Concepts, Design, Infrastructure and Uses, Kurucz CD-ROM (Cambridge: Smithsonian Astrophysical Observatory)

Landstreet, J. D. 1998, A\&A, 338, 1041

Martinet, L. 1970, A\&A, 4, 331

Michaud, G. 1980, AJ, 85, 589

Michaud, G. 1982, ApJ, 258, 349

Miles, B. M., \& Wiese, W. L. 1969, Atomic Data, 1, 1

Monier, R. 2005, A\&A, 442, 563

Moultaka, J., Ilovaisky, S. A., Prugniel, P., \& Soubiran, C. 2004, PASP, 116, 693

Napiwotzki, R., Schoenberner, D., \& Wenske, V. 1993, A\&A, 268, 653

Palacios, A., Gebran, M., Josselin, E., et al. 2010, A\&A, 516, A13

Palmer, D. R., Walker, E. N., Jones, D. H. P., \& Wallis, R. E. 1968, Royal Greenwich Observatory Bulletin, 135, 385

Pédoussaut, A., Capdeville, A., Ginestet, N., \& Carquillat, J. M. 1985, List of spectroscopic binaries from the Toulouse general catalogue (Observatoire de Toulouse)

Perruchot, S., Kohler, D., Bouchy, F., et al. 2008, in Ground-based and Airborne Instrumentation for Astronomy II, eds. I. S. McLean \& M. M. Casali, Proc. SPIE, 7014

Perryman, M. A. C., Lindegren, L., Kovalevsky, J., et al. 1997, A\&A, 323, L49

Pickering, J. C., Thorne, A. P., \& Perez, R. 2002, ApJS, 138, 247

Pietrinferni, A., Cassisi, S., Salaris, M., \& Castelli, F. 2006, ApJ, 642, 797

Preston, G. W. 1974, ARA\&A, 12, 257

R Development Core Team 2011, R: A Language and Environment for Statistical Computing, R Foundation for Statistical Computing, Vienna, Austria

Ramella, M., Böhm, C., Gerbaldi, M., \& Faraggiana, R. 1989, A\&A, 209, 233

Renson, P., \& Manfroid, J. 2009, A\&A, 498, 961

Renson, P., Gerbaldi, M., \& Catalano, F. A. 1991, A\&AS, 89, 429

Roby, S. W., \& Lambert, D. L. 1990, ApJS, 73, 67

Royer, F., Gerbaldi, M., Faraggiana, R., \& Gómez, A. E. 2002a, A\&A, 381, 105

Royer, F., Grenier, S., Baylac, M.-O., Gómez, A. E., \& Zorec, J. 2002b, A\&A, 393, 897 (RGBGZ)

Royer, F., Zorec, J., \& Gómez, A. E. 2007, A\&A, 463, 671 (RZG)

Schröder, C., \& Schmitt, J. H. M. M. 2007, A\&A, 475, 677

Schröder, C., Hubrig, S., \& Schmitt, J. H. M. M. 2008, A\&A, 484, 479

Sigut, T. A. A., \& Landstreet, J. D. 1990, MNRAS, 247, 611

Smalley, B. 2004, in The A-Star Puzzle, eds. J. Zverko, J. Žižňovský, S. J. Adelman, \& W. W. Weiss, IAU Symp., 224, 131

Stoehr, F., White, R., Smith, M., et al. 2008, in Astronomical Data Analysis Software and Systems XVII, eds. R. W. Argyle, P. S. Bunclark, \& J. R. Lewis, ASP Conf. Ser., 394, 505

Takeda, Y. 1995, PASJ, 47, 287

Torres, G., Andersen, J., \& Giménez, A. 2010, A\&ARv, 18, 67

van Leeuwen, F. 2007, A\&A, 474, 653

Varenne, O. 1999, A\&A, 341, 233

Zorec, J., \& Royer, F. 2012, A\&A, 537, A120

Zucker, S. 2003, MNRAS, 342, 1291 
Table 1. List of the 47 targets, with their derived parameters: rotational velocities, microturbulent velocity $\xi_{t}$, effective temperature, gravity, luminosity and limb-darkening coefficient $\epsilon$ in the $B$ band.

\begin{tabular}{|c|c|c|c|c|c|c|c|c|c|c|}
\hline \multirow[t]{2}{*}{ HD } & \multirow[t]{2}{*}{ SpType } & \multirow[t]{2}{*}{$\begin{array}{c}V \\
(\mathrm{mag})\end{array}$} & \multicolumn{3}{|c|}{$\begin{array}{c}v \sin i \\
\left(\mathrm{~km} \mathrm{~s}^{-1}\right)\end{array}$} & \multirow[t]{2}{*}{$\begin{array}{c}\xi_{\mathrm{t}} \\
\left(\mathrm{km} \mathrm{s}^{-1}\right)\end{array}$} & \multirow[t]{2}{*}{$\begin{array}{l}T_{\text {eff }} \\
(\mathrm{K})\end{array}$} & \multirow[t]{2}{*}{$\log g$} & \multirow[t]{2}{*}{$\log L / L_{\odot}$} & \multirow[t]{2}{*}{$\epsilon$} \\
\hline & & & RGBGZ & SYNTH & FT & & & & & \\
\hline 1439 & A0IV & 5.875 & 39 & 43.0 & $45.3_{ \pm 1.5}$ & 1.0 & 9640 & 3.75 & $2.08_{ \pm 0.04}$ & 0.6145 \\
\hline 1561 & A0Vs & 6.538 & 60 & 63.7 & $66.2 \pm 1.4$ & 1.3 & 8860 & 3.35 & $1.91_{ \pm 0.07}$ & 0.6580 \\
\hline 6530 & A1V & 5.578 & 51 & 48.8 & $103.1_{ \pm 3.0}$ & 1.6 & 9500 & 3.87 & $2.25 \pm 0.04$ & 0.6191 \\
\hline 20149 & A1Vs & 5.606 & 23 & 21.8 & $21.9_{ \pm 1.0}$ & 1.0 & 9640 & 4.04 & $2.15_{ \pm 0.06}$ & 0.6113 \\
\hline 21050 & A1V & 6.070 & 27 & 26.5 & $27.8_{ \pm 0.9}$ & 1.4 & 10420 & 4.30 & $1.62 \pm 0.03$ & 0.5781 \\
\hline 25175 & $\mathrm{~A} 0 \mathrm{~V}$ & 6.311 & 55 & 55.0 & $56.7_{ \pm 1.5}$ & 1.5 & 9920 & 3.75 & $2.09_{ \pm 0.07}$ & 0.6031 \\
\hline 28780 & A1V & 5.908 & 28 & 31.0 & $33.0_{ \pm 0.7}$ & 1.7 & 9640 & 3.86 & $1.88_{ \pm 0.05}$ & 0.6135 \\
\hline 30085 & A0IV & 6.345 & 26 & 23.0 & $24.2 \pm 0.6$ & 0.5 & 11300 & 3.95 & $2.28_{ \pm 0.07}$ & 0.5516 \\
\hline 33654 & A0V & 6.156 & 60 & 72.0 & $73.0_{ \pm 4.1}$ & 3.3 & 9220 & 2.90 & $3.63_{ \pm 0.84}$ & 0.6389 \\
\hline 39985 & A0IV & 5.971 & 28 & 28.5 & $28.2 \pm 1.2$ & 0.6 & 10240 & 3.62 & $1.83_{ \pm 0.05}$ & 0.5917 \\
\hline 40446 & A1Vs & 5.213 & 27 & 28.0 & $23.6 \pm 3.5$ & 0.5 & 9550 & 3.95 & $2.16 \pm 0.10$ & 0.6161 \\
\hline 46642 & A0Vs & 6.46 & 49 & 57.0 & $57.7_{ \pm 1.4}$ & 2.3 & 9890 & 3.89 & $1.57_{ \pm 0.11}$ & 0.6028 \\
\hline 47863 & A1V & 6.284 & 40 & 41.0 & $47.1_{ \pm 2.6}$ & 2.2 & 9520 & 3.45 & $2.34_{ \pm 0.10}$ & 0.6224 \\
\hline 50931 & $\mathrm{~A} 0 \mathrm{~V}$ & 6.274 & 65 & 75.0 & $84.0_{ \pm 5.5}$ & 1.4 & 9440 & 4.13 & $1.31_{ \pm 0.03}$ & 0.6185 \\
\hline 58142 & A1V & 4.614 & 19 & 19.0 & $18.7_{ \pm 0.4}$ & 1.7 & 9520 & 3.79 & $1.97 \pm 0.02$ & 0.6193 \\
\hline 65900 & A1V & 5.646 & 33 & 34.8 & $36.4 \pm 1.5$ & 2.0 & 9600 & 4.01 & $1.67 \pm 0.04$ & 0.6135 \\
\hline 67959 & A1V & 6.217 & 18 & 16.7 & $15.5_{ \pm 0.5}$ & 2.0 & 9310 & 3.71 & $2.03 \pm 0.07$ & 0.6296 \\
\hline 72660 & A1V & 5.799 & 9 & 7.0 & $6.5 \pm 0.4$ & 1.5 & 9640 & 4.03 & $1.57 \pm 0.03$ & 0.6116 \\
\hline 73316 & A1V & 6.542 & 33 & 32.8 & $35.2 \pm 1.1$ & 2.0 & 9830 & 4.30 & $1.49_{ \pm 0.04}$ & 0.5994 \\
\hline 83373 & A1V & 6.391 & 28 & 28.5 & $29.8 \pm 1.4$ & 1.6 & 10200 & 4.10 & $1.73_{ \pm 0.05}$ & 0.5884 \\
\hline 85504 & A0Vs & 6.02 & 27 & 25.7 & $27.6_{ \pm 2.2}$ & 1.6 & 10200 & 3.82 & $2.26 \pm 0.08$ & 0.5915 \\
\hline 89774 & A1V & 6.169 & 60 & 63.3 & $65.5 \pm 2.1$ & 2.1 & 9630 & 3.90 & $1.83_{ \pm 0.04}$ & 0.6135 \\
\hline 95418 & A1V & 2.346 & 46 & 45.7 & $46.2 \pm 1.2$ & 2.2 & 9620 & 3.89 & $1.82_{ \pm 0.00}$ & 0.6140 \\
\hline 101369 & $\mathrm{~A} 0 \mathrm{~V}$ & 6.210 & 65 & 67.5 & $70.0 \pm 3.9$ & 1.0 & 9700 & 3.82 & $1.97 \pm 0.11$ & 0.6112 \\
\hline 104181 & A1V & 5.357 & 44 & 59.0 & $55.5 \pm 1.6$ & 1.0 & 9660 & 4.00 & $1.79_{ \pm 0.02}$ & 0.6110 \\
\hline 107655 & $\mathrm{~A} 0 \mathrm{~V}$ & 6.176 & 46 & 45.0 & $47.2_{ \pm 1.4}$ & 2.0 & 9680 & 4.10 & $1.53 \pm 0.04$ & 0.6088 \\
\hline 119537 & A1V & 6.502 & 13 & 16.7 & $13.3_{ \pm 0.7}$ & 2.3 & 9260 & 4.14 & $1.54_{ \pm 0.04}$ & 0.6269 \\
\hline 127304 & A0Vs & 6.055 & 14 & 8.9 & $7.7 \pm 0.6$ & 1.5 & 10050 & 4.11 & $1.70_{ \pm 0.04}$ & 0.5939 \\
\hline 132145 & A1V & 6.506 & 15 & 13.5 & $12.7 \pm 0.6$ & 1.7 & 9680 & 4.25 & $1.59_{ \pm 0.05}$ & 0.6063 \\
\hline 133962 & A1V & 5.581 & 49 & 52.3 & $54.8 \pm 1.6$ & 1.2 & 10130 & 4.32 & $1.66 \pm 0.02$ & 0.5882 \\
\hline 145647 & A0V & 6.092 & 43 & 44.0 & $47.2 \pm 1.3$ & 0.5 & 9560 & 3.95 & $1.72 \pm 0.05$ & 0.6159 \\
\hline 145788 & A1V & 6.255 & 16 & 13.0 & $9.8 \pm 0.8$ & 2.3 & 9410 & 3.73 & $1.88_{ \pm 0.08}$ & 0.6250 \\
\hline 154228 & A1V & 5.918 & 42 & 44.8 & $45.2 \pm 1.2$ & 2.2 & 9750 & 4.20 & $1.40_{ \pm 0.02}$ & 0.6042 \\
\hline 156653 & A1V & 6.002 & 43 & 43.6 & $45.3_{ \pm 2.0}$ & 2.3 & 9270 & 3.69 & $1.82_{ \pm 0.08}$ & 0.6320 \\
\hline 158716 & A1V & 6.464 & 15 & 8.0 & $6.4 \pm 0.8$ & 2.8 & 9300 & 4.39 & $1.19_{ \pm 0.03}$ & 0.6215 \\
\hline 172167 & $\mathrm{~A} 0 \mathrm{~V}$ & 0.03 & 24 & 23.5 & $24.5 \pm 1.4$ & 1.0 & 9550 & 4.05 & $1.73_{ \pm 0.00}$ & 0.6149 \\
\hline 174567 & A0Vs & 6.634 & 15 & 13.0 & $10.5_{ \pm 0.9}$ & 1.0 & 9710 & 3.59 & $2.23 \pm 0.10$ & 0.6131 \\
\hline 176984 & A1V & 5.42 & 23 & 30.0 & $29.6 \pm 1.0$ & 2.0 & 9680 & 3.44 & $2.20_{ \pm 0.04}$ & 0.6155 \\
\hline 183534 & A1V & 5.75 & 49 & 53.0 & $51.8 \pm 5.8$ & 0.2 & 9930 & 4.24 & $1.79_{ \pm 0.02}$ & 0.5966 \\
\hline 196724 & $\mathrm{~A} 0 \mathrm{~V}$ & 4.82 & 52 & 50.5 & $51.6 \pm 2.2$ & 1.3 & 10400 & 4.18 & $1.87_{ \pm 0.06}$ & 0.5801 \\
\hline 198552 & A1Vs & 6.618 & 52 & 51.6 & $53.7 \pm 1.1$ & 3.0 & 9230 & 4.14 & $1.39_{ \pm 0.06}$ & 0.6284 \\
\hline 199095 & $\mathrm{~A} 0 \mathrm{~V}$ & 5.748 & 32 & 27.8 & $27.8 \pm 1.3$ & 0.9 & 9920 & 4.05 & $1.79_{ \pm 0.02}$ & 0.5999 \\
\hline 217186 & A1V & 6.342 & 60 & 64.0 & $66.7 \pm 2.0$ & 1.9 & 9190 & 4.00 & $1.29_{ \pm 0.04}$ & 0.6330 \\
\hline 219290 & $\mathrm{~A} 0 \mathrm{~V}$ & 6.319 & 54 & 59.0 & $61.4_{ \pm 1.9}$ & 1.5 & 9790 & 4.15 & $1.66 \pm 0.04$ & 0.6034 \\
\hline 219485 & $\mathrm{~A} 0 \mathrm{~V}$ & 5.886 & 23 & 27.0 & $27.3_{ \pm 0.8}$ & 1.2 & 9580 & 3.81 & $1.91_{ \pm 0.03}$ & 0.6165 \\
\hline 223386 & A0V & 6.328 & 33 & 33.0 & $36.8 \pm 1.2$ & 1.2 & 9890 & 4.11 & $1.62 \pm 0.07$ & 0.6001 \\
\hline 223855 & A1V & 6.292 & 60 & 62.0 & $71.5_{ \pm 4.6}$ & 1.3 & 9890 & 4.14 & $1.70_{ \pm 0.06}$ & 0.5999 \\
\hline
\end{tabular}

Notes. The spectral types and $V$ magnitudes are taken from the HIPPARcos catalog (ESA 1997). The listed $v$ sin $i$ values are: (RGBGZ) taken from Royer et al. (2002b), with an error of $\pm 10 \%$, (SYNTH) derived from the spectral synthesis detailed in Sect. 4, with a typical error of $\pm 5 \%$ and (FT) derived from the Fourier analysis of individual line profiles (Sect. 5). Errors on $\xi_{\mathrm{t}}, T_{\text {eff }}$ and $\log g$ are $\pm 0.5 \mathrm{~km} \mathrm{~s}^{-1}, \pm 125 \mathrm{~K}$, and $\pm 0.2 \mathrm{dex}$, respectively. 
F. Royer et al.: Normal A0-A1 stars with low rotational velocities. I.

Table 2. List of observations and barycentric radial velocity measurements (RV) for the target sample.

\begin{tabular}{|c|c|c|c|c|c|c|}
\hline HD & Instrument & Date & $\begin{array}{c}\# \\
\text { spectra }\end{array}$ & MJD & $S / N$ & $\begin{array}{c}\mathrm{RV} \\
\left(\mathrm{km} \mathrm{s}^{-1}\right)\end{array}$ \\
\hline 1439 & $\begin{array}{l}\text { SOPHIE } \\
\text { SOPHIE }\end{array}$ & $\begin{array}{l}2009-08-05 \\
2011-02-11\end{array}$ & $\begin{array}{l}1 \\
2\end{array}$ & $\begin{array}{l}55049.055625 \\
55603.765526\end{array}$ & $\begin{array}{l}213 \\
326\end{array}$ & $\begin{array}{r}-9.57 \pm 0.46 \\
-10.38 \pm 0.48\end{array}$ \\
\hline 1561 & $\begin{array}{l}\text { SOPHIE } \\
\text { SOPHIE }\end{array}$ & $\begin{array}{l}2009-08-05 \\
2011-02-11\end{array}$ & $\begin{array}{l}1 \\
3\end{array}$ & $\begin{array}{l}55049.042685 \\
55603.792789\end{array}$ & $\begin{array}{l}210 \\
337\end{array}$ & $\begin{array}{c}-18.44 \pm 0.88 \\
-0.04 \pm 0.92\end{array}$ \\
\hline 6530 & SOPHIE & 2009-08-05 & 3 & 55049.122963 & 387 & $10.71 \pm 2.75$ \\
\hline 20149 & $\begin{array}{l}\text { ELODIE* } \\
\text { SOPHIE } \\
\text { SOPHIE } \\
\text { SOPHIE } \\
\text { SOPHIE }\end{array}$ & $\begin{array}{l}2004-01-02 \\
2009-08-05 \\
2011-02-11 \\
2012-02-13 \\
2012-02-14\end{array}$ & $\begin{array}{l}1 \\
3 \\
2 \\
2 \\
2\end{array}$ & $\begin{array}{l}53006.871601 \\
55049.086474 \\
55603.834201 \\
55970.794641 \\
55971.764421\end{array}$ & $\begin{array}{l}106 \\
399 \\
356 \\
341 \\
370\end{array}$ & $\begin{array}{c}-9.01 \pm 0.16 \\
-12.11 \pm 0.16 \\
-12.32 \pm 0.16 \\
-10.70 \pm 0.16 \\
-10.88 \pm 0.16\end{array}$ \\
\hline 21050 & $\begin{array}{l}\text { SOPHIE } \\
\text { SOPHIE } \\
\text { SOPHIE }\end{array}$ & $\begin{array}{l}2009-08-05 \\
2011-02-11 \\
2012-02-13 \\
\end{array}$ & $\begin{array}{l}1 \\
2 \\
2\end{array}$ & $\begin{array}{l}55049.104537 \\
55603.856759 \\
55970.773571\end{array}$ & $\begin{array}{c}226 \\
69 \\
328 \\
\end{array}$ & $\begin{array}{l}-1.10 \pm 0.23 \\
-1.08 \pm 0.44 \\
-0.89 \pm 0.24\end{array}$ \\
\hline 25175 & $\begin{array}{l}\text { SOPHIE } \\
\text { SOPHIE }\end{array}$ & $\begin{array}{l}2011-02-11 \\
2012-02-13\end{array}$ & $\begin{array}{l}1 \\
3\end{array}$ & $\begin{array}{l}55603.817766 \\
55970.818221\end{array}$ & $\begin{array}{l}222 \\
308\end{array}$ & $\begin{array}{l}31.62 \pm 0.76 \\
31.33 \pm 0.73\end{array}$ \\
\hline 28780 & SOPHIE & 2012-02-13 & 2 & 55970.847066 & 340 & $-14.19 \pm 0.29$ \\
\hline 30085 & SOPHIE & 2012-02-13 & 3 & 55970.874525 & 316 & $8.27 \pm 0.20$ \\
\hline 33654 & SOPHIE & $2012-02-13$ & 2 & 55970.904277 & 180 & $-3.12 \pm 1.10$ \\
\hline 39985 & ELODIE* & 1995-12-20 & 1 & 50071.994159 & 138 & $13.47 \pm 0.24$ \\
\hline 40446 & ELODIE* & 1999-12-20 & 1 & 51532.843002 & 160 & $47.80 \pm 0.20$ \\
\hline 46642 & $\begin{array}{l}\text { ELODIE* } \\
\text { SOPHIE }\end{array}$ & $\begin{array}{l}2003-01-15 \\
2012-02-14\end{array}$ & $\begin{array}{l}1 \\
3\end{array}$ & $\begin{array}{l}52654.978756 \\
55971.822326\end{array}$ & $\begin{array}{l}190 \\
332\end{array}$ & $\begin{array}{l}31.62 \pm 0.81 \\
38.41 \pm 0.78\end{array}$ \\
\hline 47863 & SOPHIE & 2012-02-14 & 3 & 55971.789437 & 365 & $23.80 \pm 0.52$ \\
\hline 50931 & SOPHIE & 2012-02-14 & 3 & 55971.859687 & 345 & $42.06 \pm 1.46$ \\
\hline 58142 & $\begin{array}{l}\text { ELODIE* } \\
\text { ELODIE* } \\
\text { ELODIE* } \\
\text { ELODIE* }\end{array}$ & $\begin{array}{l}1998-01-28 \\
2004-01-03 \\
2005-02-02 \\
2005-02-03\end{array}$ & $\begin{array}{l}1 \\
1 \\
1 \\
1\end{array}$ & $\begin{array}{l}50841.980507 \\
53007.964181 \\
53403.947654 \\
53404.944216\end{array}$ & $\begin{array}{l}172 \\
300 \\
270 \\
292\end{array}$ & $\begin{array}{l}26.94 \pm 0.12 \\
26.76 \pm 0.12 \\
26.90 \pm 0.12 \\
26.66 \pm 0.12\end{array}$ \\
\hline 65900 & ELODIE* & $1995-12-21$ & 1 & 50072.040836 & 107 & $43.06 \pm 0.36$ \\
\hline 67959 & ELODIE & 2005-04-21 & 1 & 53481.846784 & 106 & $23.74 \pm 0.09$ \\
\hline 72660 & $\begin{array}{l}\text { ELODIE* } \\
\text { ELODIE* } \\
\text { ELODIE* } \\
\text { ELODIE* }\end{array}$ & $\begin{array}{l}1997-03-20 \\
2004-01-03 \\
2004-01-04 \\
2004-04-11\end{array}$ & $\begin{array}{l}1 \\
1 \\
1 \\
1\end{array}$ & $\begin{array}{l}50527.808905 \\
53007.074899 \\
53009.017029 \\
53106.829269\end{array}$ & $\begin{array}{c}64 \\
118 \\
152 \\
192\end{array}$ & $\begin{array}{l}3.22 \pm 0.03 \\
4.28 \pm 0.06 \\
4.68 \pm 0.06 \\
4.74 \pm 0.07\end{array}$ \\
\hline 73316 & $\begin{array}{l}\text { SOPHIE } \\
\text { SOPHIE }\end{array}$ & $\begin{array}{l}2012-02-13 \\
2012-02-14\end{array}$ & $\begin{array}{l}3 \\
3\end{array}$ & $\begin{array}{l}55970.946798 \\
55971.894159\end{array}$ & $\begin{array}{l}146 \\
335\end{array}$ & $\begin{array}{l}27.68 \pm 0.34 \\
28.02 \pm 0.33\end{array}$ \\
\hline 83373 & SOPHIE & 2012-02-14 & 4 & 55971.999699 & 292 & $26.58 \pm 0.24$ \\
\hline 85504 & $\begin{array}{l}\text { ELODIE* } \\
\text { SOPHIE } \\
\text { SOPHIE }\end{array}$ & $\begin{array}{l}1996-04-24 \\
2012-02-13 \\
2012-02-14 \\
\end{array}$ & $\begin{array}{l}1 \\
2 \\
4\end{array}$ & $\begin{array}{l}50197.825920 \\
55971.001111 \\
55971.960573\end{array}$ & $\begin{array}{c}114 \\
63 \\
289\end{array}$ & $\begin{array}{l}103.76 \pm 0.24 \\
104.03 \pm 0.32 \\
103.70 \pm 0.24\end{array}$ \\
\hline 89774 & $\begin{array}{l}\text { ELODIE } \\
\text { SOPHIE }\end{array}$ & $\begin{array}{l}2005-04-22 \\
2012-02-14\end{array}$ & $\begin{array}{l}1 \\
3\end{array}$ & $\begin{array}{l}53482.912204 \\
55971.926975\end{array}$ & $\begin{array}{l}102 \\
293\end{array}$ & $\begin{array}{l}13.28 \pm 0.94 \\
12.18 \pm 0.85\end{array}$ \\
\hline 95418 & $\begin{array}{c}\text { ELODIE* } \\
\text { ELODIE* } \\
\text { ELODIE* } \\
\text { SOPHIE }\end{array}$ & $\begin{array}{l}1997-02-20 \\
2004-03-10 \\
2005-02-04 \\
2011-02-11\end{array}$ & $\begin{array}{l}1 \\
1 \\
1 \\
5\end{array}$ & $\begin{array}{l}50499.547959 \\
53074.002745 \\
53405.056783 \\
55604.191435\end{array}$ & $\begin{array}{l}170 \\
232 \\
283 \\
375\end{array}$ & $\begin{array}{l}-13.52 \pm 0.66 \\
-13.73 \pm 0.50 \\
-13.89 \pm 0.53 \\
-13.67 \pm 0.51\end{array}$ \\
\hline 101369 & ELODIE & 2005-04-21 & 2 & 53481.910739 & 182 & $7.70 \pm 1.79$ \\
\hline 104181 & $\begin{array}{l}\text { ELODIE* } \\
\text { SOPHIE }\end{array}$ & $\begin{array}{l}2004-04-26 \\
2012-02-14\end{array}$ & $\begin{array}{l}1 \\
3\end{array}$ & $\begin{array}{l}53121.940547 \\
55972.076979\end{array}$ & $\begin{array}{l}209 \\
299\end{array}$ & $\begin{array}{l}-1.29 \pm 0.84 \\
-0.34 \pm 0.73\end{array}$ \\
\hline 107655 & ELODIE* & 2004-04-08 & 1 & 53103.058789 & 243 & $-2.74 \pm 0.59$ \\
\hline
\end{tabular}

Notes. Asterisks, following the instrument name, indicate the spectra have been retrieved from the corresponding archive. Spectra observed the same night were combined into one spectrum. The number of input spectra, the modified Julian date (MJD) at the center of the exposure(s) and the combined signal-to-noise $(\mathrm{S} / \mathrm{N})$ are also given. 
Table 2. continued.

\begin{tabular}{|c|c|c|c|c|c|c|}
\hline HD & Instrument & Date & $\begin{array}{c}\# \\
\text { spectra }\end{array}$ & MJD & $S / N$ & $\begin{array}{c}\mathrm{RV} \\
\left(\mathrm{km} \mathrm{s}^{-1}\right)\end{array}$ \\
\hline & SOPHIE & 2012-02-14 & 3 & 55972.034603 & 338 & $-3.49 \pm 0.54$ \\
\hline 119537 & $\begin{array}{l}\text { ELODIE } \\
\text { SOPHIE }\end{array}$ & $\begin{array}{l}2006-06-02 \\
2012-02-14\end{array}$ & $\begin{array}{l}1 \\
7\end{array}$ & $\begin{array}{l}53888.856696 \\
55972.175921\end{array}$ & $\begin{array}{l}105 \\
254\end{array}$ & $\begin{array}{c}54.81 \pm 0.08 \\
-62.46 \pm 0.07\end{array}$ \\
\hline $\begin{array}{l}127304 \\
132145\end{array}$ & $\begin{array}{l}\text { ELODIE } \\
\text { ELODIE } \\
\text { SOPHIE }\end{array}$ & $\begin{array}{l}2005-04-21 \\
2005-04-22 \\
2012-02-14\end{array}$ & $\begin{array}{l}1 \\
2 \\
4\end{array}$ & $\begin{array}{l}53481.985365 \\
53482.066887 \\
55972.113021\end{array}$ & $\begin{array}{l}223 \\
219 \\
277 \\
\end{array}$ & $\begin{array}{c}-22.30 \pm 0.11 \\
-9.98 \pm 0.07 \\
-9.73 \pm 0.07\end{array}$ \\
\hline 133962 & $\begin{array}{l}\text { ELODIE } \\
\text { SOPHIE } \\
\text { SOPHIE } \\
\end{array}$ & $\begin{array}{l}2005-04-22 \\
2011-02-11 \\
2012-02-14 \\
\end{array}$ & $\begin{array}{l}1 \\
2 \\
3 \\
\end{array}$ & $\begin{array}{l}53482.131317 \\
55604.224086 \\
55972.058538 \\
\end{array}$ & $\begin{array}{l}201 \\
215 \\
329 \\
\end{array}$ & $\begin{array}{l}-12.63 \pm 0.73 \\
-13.29 \pm 0.73 \\
-13.11 \pm 0.72 \\
\end{array}$ \\
\hline 145647 & $\begin{array}{l}\text { ELODIE } \\
\text { ELODIE }\end{array}$ & $\begin{array}{l}2006-05-31 \\
2006-06-03 \\
\end{array}$ & $\begin{array}{l}2 \\
1 \\
\end{array}$ & $\begin{array}{l}53886.908366 \\
53889.067390 \\
\end{array}$ & $\begin{array}{l}107 \\
156 \\
\end{array}$ & $\begin{array}{l}-15.12 \pm 0.85 \\
-15.75 \pm 0.58\end{array}$ \\
\hline 145788 & ELODIE & 2006-06-01 & 2 & 53887.968344 & 128 & $-13.05 \pm 0.05$ \\
\hline 154228 & $\begin{array}{l}\text { ELODIE* } \\
\text { ELODIE* }\end{array}$ & $\begin{array}{l}1999-06-05 \\
1999-06-06 \\
\end{array}$ & $\begin{array}{l}1 \\
1 \\
\end{array}$ & $\begin{array}{l}51334.988236 \\
51335.970245 \\
\end{array}$ & $\begin{array}{l}163 \\
244 \\
\end{array}$ & $\begin{array}{l}-31.10 \pm 0.50 \\
-31.66 \pm 0.47 \\
\end{array}$ \\
\hline 156653 & $\begin{array}{l}\text { ELODIE* } \\
\text { SOPHIE }\end{array}$ & $\begin{array}{l}1996-04-26 \\
2009-08-05 \\
\end{array}$ & $\begin{array}{l}1 \\
3 \\
\end{array}$ & $\begin{array}{l}50199.123847 \\
55048.835204 \\
\end{array}$ & $\begin{array}{l}113 \\
294 \\
\end{array}$ & $\begin{array}{l}2.37 \pm 0.47 \\
4.24 \pm 0.46\end{array}$ \\
\hline 158716 & ELODIE & 2006-06-02 & 2 & 53888.924720 & 117 & $-25.44 \pm 0.03$ \\
\hline 174567 & $\begin{array}{l}\text { ELODIE* } \\
\text { ELODIE* } \\
\text { ELODIE* } \\
\text { ELODIE* } \\
\text { ELODIE* } \\
\text { ELODIE* } \\
\text { ELODIE* } \\
\text { ELODIE* } \\
\text { ELODIE* } \\
\text { ELODIE* } \\
\text { ELODIE* } \\
\text { ELODIE* } \\
\text { ELODIE* } \\
\text { ELODIE* } \\
\text { ELODIE* } \\
\text { ELODIE* } \\
\text { SOPHIE* } \\
\text { SOPHIE* } \\
\text { SOPHIE* } \\
\text { SOPHIE* } \\
\text { SOPHIE* } \\
\text { SOPHIE* } \\
\text { SOPHIE* } \\
\text { SOPHIE* } \\
\text { SOPHIE* } \\
\text { SOPHIE* } \\
\text { ELODIE } \\
\text { SOPHIE }\end{array}$ & $\begin{array}{l}2004-02-28 \\
2004-03-01 \\
2004-03-27 \\
2004-03-29 \\
2004-08-30 \\
2004-08-31 \\
2004-09-23 \\
2004-09-24 \\
2004-09-25 \\
2004-11-05 \\
2004-11-06 \\
2004-11-07 \\
2004-11-09 \\
2005-04-20 \\
2005-05-24 \\
2005-05-25 \\
2006-09-08 \\
2009-09-28 \\
2009-09-29 \\
2009-09-30 \\
2009-10-06 \\
2009-10-07 \\
2009-10-08 \\
2009-10-09 \\
2009-10-10 \\
2009-10-11 \\
2006-05-31 \\
2009-08-05\end{array}$ & $\begin{array}{l}1 \\
1 \\
1 \\
2 \\
2 \\
2 \\
2 \\
2 \\
2 \\
2 \\
1 \\
2 \\
1 \\
2 \\
2 \\
1 \\
1 \\
4 \\
1 \\
1 \\
1 \\
2 \\
1 \\
1 \\
3 \\
1 \\
2 \\
3\end{array}$ & $\begin{array}{l}53063.215076 \\
53065.215871 \\
53091.176158 \\
53093.148334 \\
53247.816155 \\
53248.796281 \\
53271.772018 \\
53272.771336 \\
53273.769548 \\
53314.738914 \\
53315.726548 \\
53316.740124 \\
53318.732878 \\
53480.107426 \\
53514.082351 \\
53515.036852 \\
53987.400894 \\
55102.762360 \\
55103.761169 \\
55104.763217 \\
55110.777199 \\
55111.751158 \\
55112.772696 \\
55113.756793 \\
55114.744915 \\
55115.742314 \\
53887.000591 \\
55048.871358\end{array}$ & $\begin{array}{l}266 \\
288 \\
275 \\
357 \\
378 \\
355 \\
317 \\
320 \\
347 \\
296 \\
246 \\
251 \\
152 \\
309 \\
301 \\
254 \\
109 \\
1055 \\
467 \\
422 \\
386 \\
443 \\
438 \\
405 \\
571 \\
300 \\
119 \\
355\end{array}$ & $\begin{array}{l}-13.91 \pm 0.19 \\
-13.82 \pm 0.20 \\
-13.99 \pm 0.20 \\
-13.91 \pm 0.19 \\
-13.68 \pm 0.19 \\
-13.62 \pm 0.19 \\
-13.77 \pm 0.19 \\
-13.50 \pm 0.19 \\
-13.60 \pm 0.20 \\
-13.52 \pm 0.19 \\
-13.86 \pm 0.18 \\
-13.49 \pm 0.20 \\
-14.01 \pm 0.20 \\
-13.89 \pm 0.19 \\
-13.79 \pm 0.19 \\
-13.94 \pm 0.20 \\
-14.07 \pm 0.21 \\
-13.81 \pm 0.19 \\
-13.81 \pm 0.19 \\
-13.72 \pm 0.19 \\
-13.69 \pm 0.19 \\
-13.76 \pm 0.19 \\
-13.68 \pm 0.19 \\
-13.66 \pm 0.19 \\
-13.71 \pm 0.19 \\
-13.83 \pm 0.19 \\
-14.18 \pm 0.05 \\
-10.84 \pm 0.05\end{array}$ \\
\hline 176984 & $\begin{array}{l}\text { ELODIE } \\
\text { SOPHIE }\end{array}$ & $\begin{array}{l}2006-06-02 \\
2009-08-05 \\
\end{array}$ & $\begin{array}{l}1 \\
3 \\
\end{array}$ & $\begin{array}{l}53888.998090 \\
55048.903823 \\
\end{array}$ & $\begin{array}{l}153 \\
402 \\
\end{array}$ & $\begin{array}{l}-47.68 \pm 0.29 \\
-43.75 \pm 0.24\end{array}$ \\
\hline 183534 & ELODIE & 2006-06-01 & 1 & 53887.075317 & 148 & $9.88 \pm 0.63$ \\
\hline 196724 & $\begin{array}{l}\text { ELODIE* } \\
\text { ELODIE }\end{array}$ & $\begin{array}{l}2004-11-12 \\
2006-06-02 \\
\end{array}$ & $\begin{array}{l}1 \\
1 \\
\end{array}$ & $\begin{array}{l}53321.779634 \\
53888.083812 \\
\end{array}$ & $\begin{array}{l}301 \\
156 \\
\end{array}$ & $\begin{array}{l}-21.97 \pm 0.64 \\
-18.31 \pm 0.52 \\
\end{array}$ \\
\hline 198552 & SOPHIE & 2009-08-05 & 2 & 55048.952500 & 314 & $5.47 \pm 0.69$ \\
\hline 199095 & $\begin{array}{l}\text { ELODIE* } \\
\text { SOPHIE }\end{array}$ & $\begin{array}{l}2005-10-14 \\
2009-08-05\end{array}$ & $\begin{array}{l}1 \\
3\end{array}$ & $\begin{array}{l}53657.768627 \\
55048.925880\end{array}$ & $\begin{array}{l}168 \\
325\end{array}$ & $\begin{array}{c}-24.99 \pm 0.21 \\
3.49 \pm 0.23\end{array}$ \\
\hline 217186 & SOPHIE & 2009-08-05 & 3 & 55048.980456 & 287 & $18.67 \pm 1.34$ \\
\hline 219290 & SOPHIE & 2009-08-05 & 2 & 55049.003953 & 253 & $-16.88 \pm 0.83$ \\
\hline 219485 & SOPHIE & 2009-08-05 & 1 & 55049.016284 & 239 & $-4.48 \pm 0.21$ \\
\hline 223386 & SOPHIE & 2009-08-05 & 1 & 55049.027685 & 235 & $-18.57 \pm 0.34$ \\
\hline 223855 & SOPHIE & 2009-08-05 & 1 & 55049.067083 & 225 & $3.68 \pm 1.05$ \\
\hline
\end{tabular}


F. Royer et al.: Normal A0-A1 stars with low rotational velocities. I.

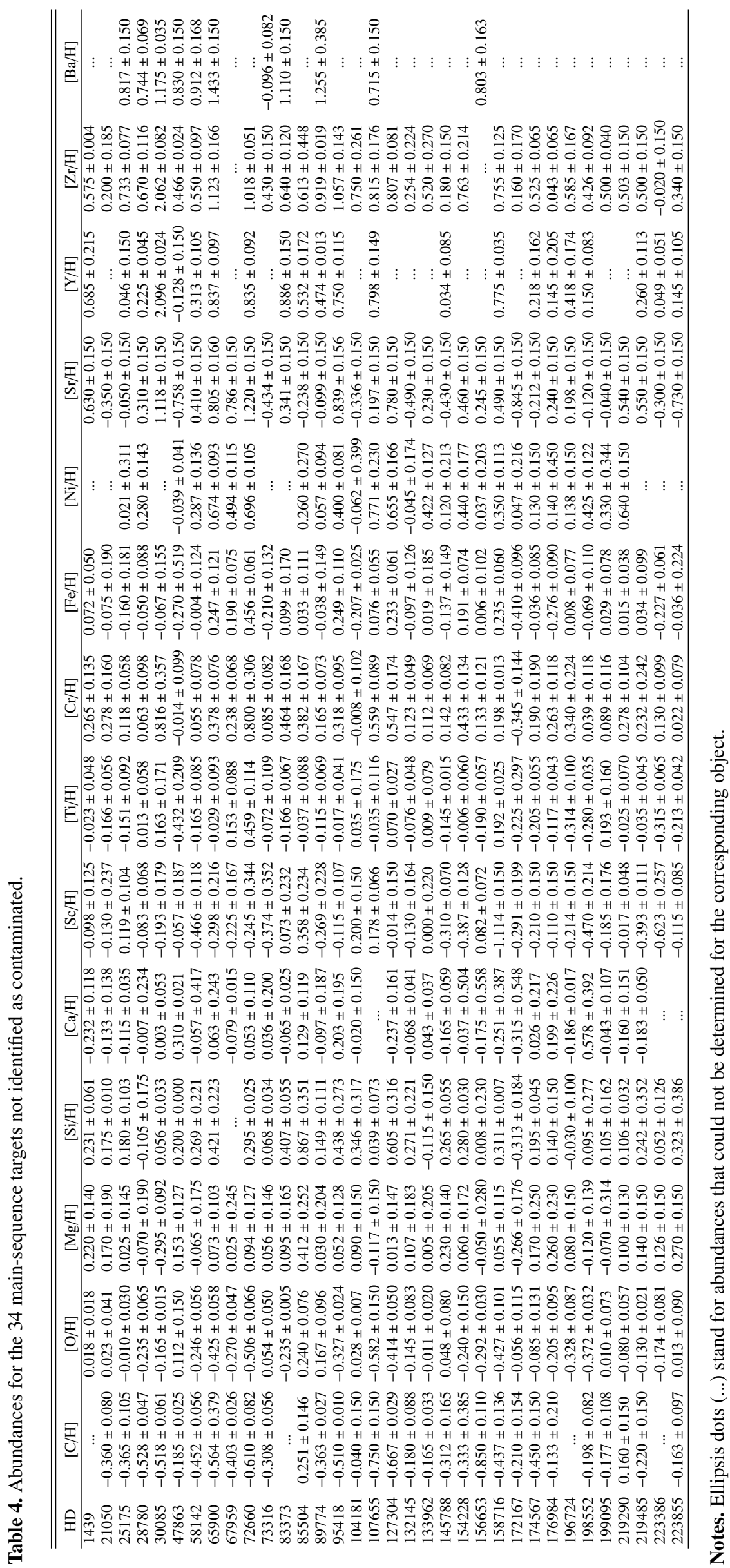


Table 5. Probabilities to be CP given by the four different criteria.

\begin{tabular}{lccccc}
\hline \hline HD & $p_{10}$ & $f_{10}$ & $p_{14}$ & $f_{14}$ & Classification \\
\hline 1439 & 0.827 & 1 & 0.342 & 0 & uncertain \\
21050 & 0.107 & 0 & 0.012 & 0 & normal \\
25175 & 0.073 & 0 & 0.035 & 0 & normal \\
28780 & 0.287 & 0 & 0.348 & 0 & normal \\
30085 & 0.986 & 1 & 0.983 & 1 & CP \\
47863 & 0.004 & 0 & 0.001 & 0 & normal \\
58142 & 0.423 & 0 & 0.400 & 0 & normal \\
65900 & 0.999 & 1 & 0.994 & 1 & CP \\
67959 & 0.960 & 1 & 0.945 & 1 & CP \\
72660 & 0.999 & 1 & 1.000 & 1 & CP \\
73316 & 0.014 & 0 & 0.010 & 0 & normal \\
83373 & 0.854 & 1 & 0.640 & 1 & CP \\
85504 & 0.428 & 0 & 0.085 & 0 & normal \\
89774 & 0.166 & 0 & 0.051 & 0 & normal \\
95418 & 0.980 & 1 & 0.983 & 1 & CP \\
104181 & 0.036 & 0 & 0.010 & 0 & normal \\
107655 & 0.884 & 1 & 0.940 & 1 & CP \\
127304 & 0.997 & 1 & 0.999 & 1 & CP \\
132145 & 0.008 & 0 & 0.005 & 0 & normal \\
133962 & 0.393 & 0 & 0.229 & 0 & normal \\
145788 & 0.012 & 0 & 0.001 & 0 & normal \\
154228 & 0.899 & 1 & 0.799 & 1 & CP \\
156653 & 0.244 & 0 & 0.411 & 0 & normal \\
158716 & 0.756 & 1 & 0.916 & 1 & CP \\
172167 & 0.001 & 0 & 0.027 & 0 & normal \\
174567 & 0.076 & 0 & 0.042 & 0 & normal \\
176984 & 0.087 & 0 & 0.054 & 0 & normal \\
196724 & 0.323 & 0 & 0.312 & 0 & normal \\
198552 & 0.111 & 0 & 0.199 & 0 & normal \\
199095 & 0.261 & 0 & 0.189 & 0 & normal \\
219290 & 0.849 & 1 & 0.349 & 0 & uncertain \\
219485 & 0.637 & 0 & 0.402 & 0 & probably normal \\
223386 & 0.014 & 0 & 0.028 & 0 & normal \\
223855 & 0.018 & 0 & 0.001 & 0 & normal \\
\hline & & & & & \\
\hline
\end{tabular}

Notes. Classification is deduced from the combination of four probabilities. 
F. Royer et al.: Normal A0-A1 stars with low rotational velocities. I.

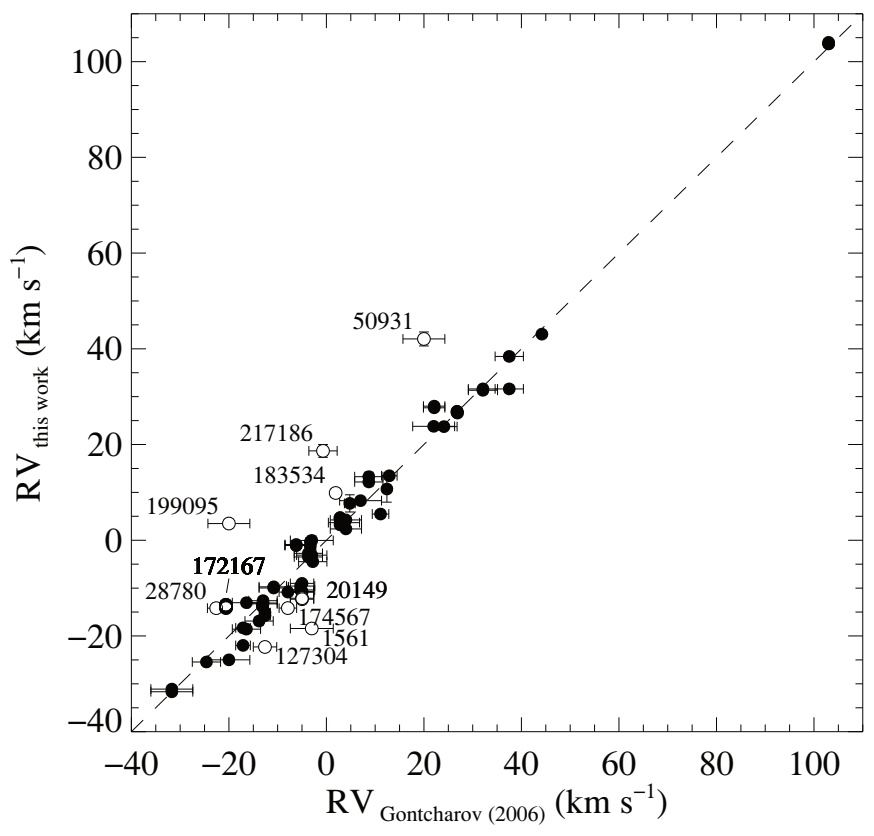

Fig. A.1. Comparison of radial velocities between individual values from Table 2 and the compilation from Gontcharov (2006). The error bars are the internal errors and the dashed line is the one-to-one relation. The outliers are indicated by open symbols, together with their HD number.

\section{Appendix A: Comments on individual stars}

All our targets are part of the sample studied by Dworetsky (1974), and 25 of them are analyzed by Ramella et al. (1989). In their paper, Ramella et al. (1989) derive $v \sin i$ from Fourier profile analysis and flag the stars according to the agreement between the observed profile and a theoretical rotation profile ("1" when agreeing, "0" when disagreeing, in their Table 3). Stars labeled as "0" by Ramella et al. are checked out with our data, and the Fourier profiles are plotted in Fig. A.3.

Gontcharov (2006) published a compilation of radial velocities and our individual measurements are compared with literature data. The 44 stars in common are plotted in Fig. A.1. The Gaussian fit of the histogram of radial velocity differences gives a standard deviation $\sigma=2 \mathrm{~km} \mathrm{~s}^{-1}$. Ten stars show differences larger than $3 \sigma$. They are indicated in Fig. A.1. The three stars of our sample (HD 40446, HD 119537 and HD 176984), that are not present in Gontcharov (2006), are already detected as binaries in our data.

HD 1439 could not be undoubtedly classified as a normal nor CP star, the derived memberships based on 10 and 14 elements giving contradictory results (Table 5). It moreover lies in the "uncertain" zone in Fig. 9. The derived abundances in oxygen and magnesium are high enough to make it classified as a normal star using all 14 elements whereas the remaining pattern $(\mathrm{Si}, \mathrm{Ca}$, $\mathrm{Sc}, \mathrm{Cr}, \mathrm{Fe}, \mathrm{Sr}, \mathrm{Y}$ and $\mathrm{Zr}$ ) is rather similar to a $\mathrm{CP}$ star. Its radial velocity observed 18 months apart does not show significant variation.

HD 1561 is variable in radial velocity, from our two observations as well as compared with Gontcharov (2006) who gives a radial velocity of $-3 \mathrm{~km} \mathrm{~s}^{-1}$ (Fig. A.1).

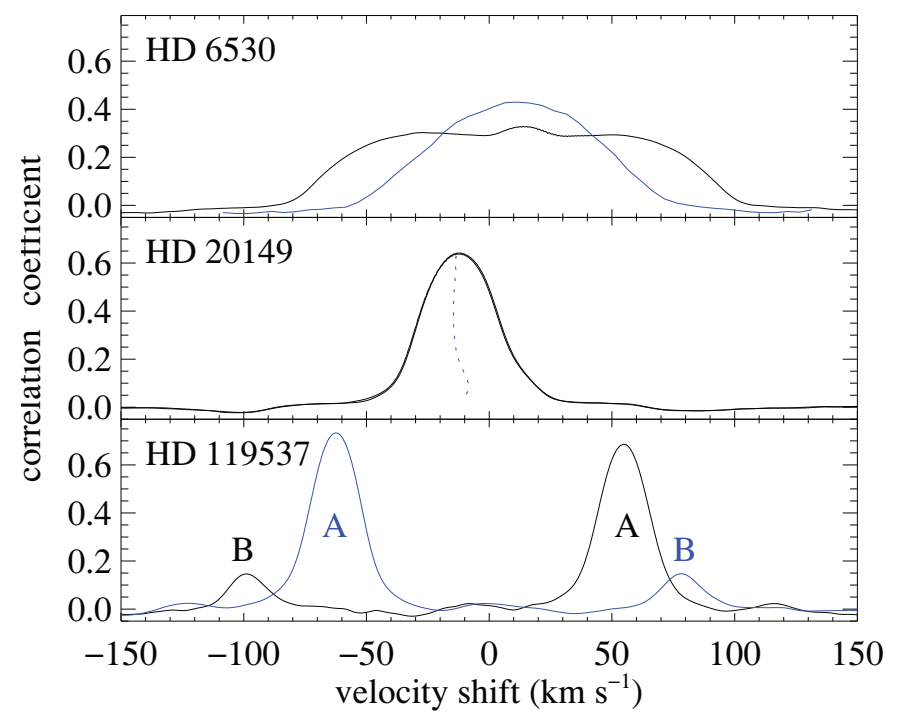

Fig. A.2. Cross-correlation functions for three suspected binary stars. Top panel: the CCF of the observed spectrum is overplotted to the one observed by Grenier et al. (1999). Middle panel: the bisector of the CCF is displayed (dotted line) with a velocity scale enhanced by a factor 3, for the sake of clarity. Bottom panel: the two components in the composite CCF are labeled as " $\mathrm{A}$ " and "B".

HD 6530 is detected as a spectroscopic binary from its CCF (Fig. 2). The large difference in the $v \sin i$ measured using the spectral synthesis and the FT (Table 1) is due to the composite spectrum. It has already been observed by Grenier et al. (1999) who derive the radial velocity and their spectrum is also used by Royer et al. (2002a) to derive the $v \sin i$. The CCF from this spectrum is overplotted in Fig. A.2 (black solid line) to emphasize the binary nature of the star.

HD 20149 is flagged as a suspected binary due to the asymmetry of the CCF. The bisector is displayed in Fig. A.2. The star is labeled as variable in radial velocity in Gontcharov (2006), and the published value $\left(-5 \mathrm{~km} \mathrm{~s}^{-1}\right)$ is also significantly different from our determinations (Fig. A.1).

HD 21050 is found to have a large projected rotational velocity by Dworetsky (1974) $\left(v \sin i=60 \mathrm{~km} \mathrm{~s}^{-1}\right)$ but all other determinations are very similar to our result (Palmer et al. 1968; Abt \& Morrell 1995; Royer et al. 2002b). Moreover, no spectral variation is detected in our high signal-to-noise observations, collected three years apart.

HD 25175 is suspected by Ramella et al. (1989) to be a spectroscopic binary due to the large broadening and the disagreement the observed profile and a theoretical rotational profile. Their determination of $v \sin i$, in good agreement with ours, is much higher than the value derived by Dworetsky $(1974)\left(\leq 40 \mathrm{~km} \mathrm{~s}^{-1}\right)$. Our Fourier profiles are plotted in Fig. A.3a and the agreement with the rotational profile is very good, suggesting that the broadening is dominated by rotation. Also both our spectra, observed one year apart, do not show any sign of radial velocity variation.

HD 28780 has a $v \sin i$ significantly different from what Ramella et al. (1989) find $\left(41.3 \mathrm{~km} \mathrm{~s}^{-1}\right)$. The radial velocity 


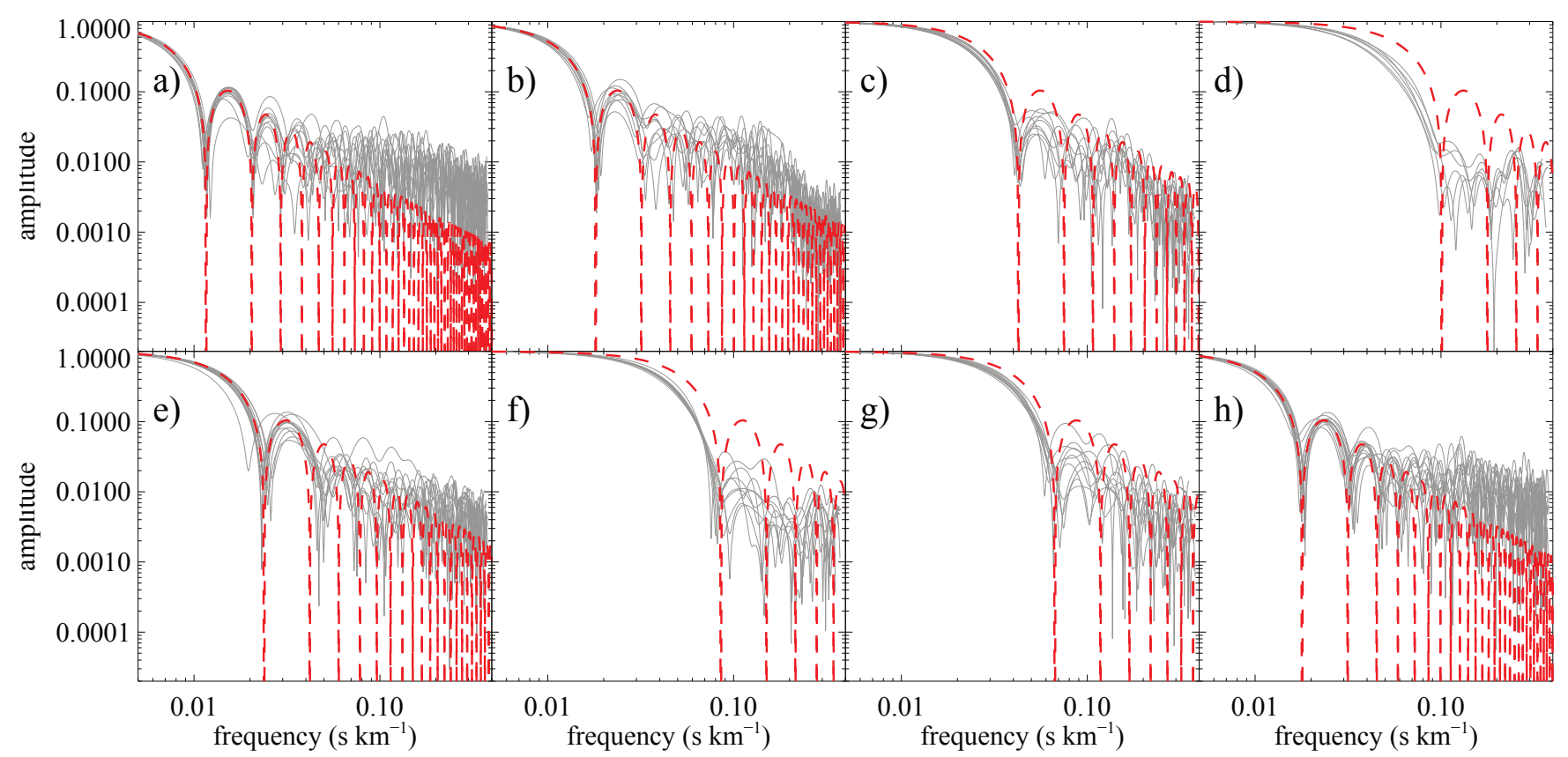

Fig. A.3. Individual line FT (solid lines) and theoretical rotation profile corresponding to the average $v \sin i$ (thick dashed line) for the stars flagged as " 0 " by Ramella et al. (1989): a) HD 25175, b) HD 65900, c) HD 67959, d) HD 72660, e) HD 85504, f) HD 127304, g) HD 145788, h) HD 223386.

is moreover significantly different from the value published by Gontcharov (2006): -22.6 $\mathrm{km} \mathrm{s}^{-1}$ (Fig. A.1). Although no evidence of binarity is detected in our single observation, these differences suggest that this object could be a spectroscopic binary.

HD 30085 is discarded from $v \sin i$ measurement by Ramella et al. (1989) because of asymmetric line profiles. In our classification, it falls in the CP group and is newly detected as chemically peculiar.

HD 33654 is identified as a giant star from both its surface gravity and its luminosity (Table 1), which disagrees with its luminosity class: A0V. It was previously classified as a B9III star (Palmer et al. 1968). It is indicated as an Ap Si star in Renson \& Manfroid (2009).

HD 39985 is an outlier in our luminosity comparison (Fig. 3) and therefore suspected to be a binary star.

HD 40446 is flagged as a spectroscopic binary by Dworetsky (1974). It is part of the sample studied by Royer et al. (2002b) who determined an uncertain $v \sin i$ with a high external error $\left(v \sin i=27: \pm 5 \mathrm{~km} \mathrm{~s}^{-1}\right)$ due to a large dispersion in the values derived from individual lines. Its binary nature is confirmed by the shape of its CCF in Fig. 2.

HD 46642 is suspected to be a photometric variable star (Kukarkin et al. 1981). Our radial velocity measurements reveal a variation hence this star is suspected to be a binary.

HD 47863 shows a significant difference between our measurements of $v \sin i$, using spectral synthesis and Fourier profile. This object is however used as a reference star in speckle observations by Aristidi et al. (1997), suggesting that it is a single star.

HD 50931 is detected as a spectroscopic binary from the distorted shape of its CCF. The radial velocity from Gontcharov (2006) is moreover very different from our result: $20 \mathrm{~km} \mathrm{~s}^{-1}$ (Fig. A.1). The large difference in the $v \sin i$ measured using the spectral synthesis and the FT (75 and $84 \mathrm{~km} \mathrm{~s}^{-1}$ in Table 1, respectively) is due to the composite spectrum.

HD 58142 is found to be a hot Am star by Adelman (1994), which disagrees with our classification. This object lies in the tail of the distribution of memberships (Table 5 and Fig. 8) and could have been wrongly assigned to the "normal" group.

HD 65900 is suspected by Ramella et al. (1989) to be a spectroscopic binary due to the large broadening and the disagreement between the observed profile and a theoretical rotational profile. This disagreement is not seen in our data (Fig. A.3b). In our classification, it falls in the $\mathrm{CP}$ group and is newly detected as chemically peculiar.

HD 67959 is found to disagree with a rotation profile by Ramella et al. (1989), but this is very probably due to its low $v \sin i$ and the fact that instrumental broadening is not negligible. In our data (Fig. A.3c), the main lobe of the FT in observed profiles is well fitted by the theoretical rotation profile. In our classification, it falls in the $\mathrm{CP}$ group and is newly detected as chemically peculiar.

HD 72660 is a hot Am star (Varenne 1999), which is consistent with our classification. Its low $v \sin i$ makes the Fourier profiles dominated by the instrumental profile, both in Ramella et al. (1989) and in Fig. A.3d. It is listed in Table 3 as variable in radial 
Table A.1. Comparison of Sc and Sr abundances of HD 107655 with Gebran et al. (2008).

\begin{tabular}{lcc}
\hline \hline Element / line & This study & Gebran et al. (2008) \\
\hline$[\mathrm{Sc} / \mathrm{H}]$ & $0.178 \pm 0.066$ & $-0.2 \pm 0.11$ \\
$4314.083 \AA$ & $\ldots$ & 2.55 \\
$4320.732 \AA$ & $\ldots$ & 3.02 \\
$4324.996 \AA$ & $\ldots$ & 2.81 \\
$4374.457 \AA$ & 3.374 & 3.14 \\
$5657.896 \AA$ & 3.243 & 3.13 \\
\hline$[\mathrm{Sr} / \mathrm{H}]$ & $0.197 \pm 0.150$ & $-0.23 \pm 0.20$ \\
$4077.709 \AA$ & $\ldots$ & 2.29 \\
$4215.520 \AA$ & 3.127 & 3.08 \\
\hline
\end{tabular}

Notes. The mean abundances, relative to the Sun, are given, as well as the absolute abundances for each spectral line. Ellipsis dots (...) indicate when a spectral line has not been used.

velocity, but the variation remains very small. Landstreet (1998) detects an asymmetry in the spectral lines that is attributed to a depth-dependent velocity field.

HD 85504 is flagged by Ramella et al. (1989) as showing a profile disagreeing with a rotational broadening. In our data however (Fig. A.3e), the agreement with the theoretical rotational profile is good. This object is flagged in Renson \& Manfroid (2009) as an Ap Mn star, and manganese is not part of our analyzed chemical species. In Adelman \& Pintado (1997), it only appears slightly metal rich compared to other superficially normal stars with similar effective temperature. This object is moreover known as high spacial velocity (Martinet 1970; Altmann \& de Boer 2000) which may be a runaway star. It is a suspected variable star (Kukarkin et al. 1981).

HD 95418 is an Am star (Adelman et al. 2011; Hill 1995) and our classification agrees with these results.

HD 101369 is suspected to be a spectroscopic binary from the shape of its CCF (Fig. 2).

HD 107655 belongs to the open cluster Coma Ber. Gebran et al. (2008) derived abundances, using the same method, and their values are in good agreement with our determinations, with larger differences for Sc and Sr. The dispersion in the Sc and $\mathrm{Sr}$ abundances derived by Gebran et al. (2008) is high; they include more lines than in this study, especially lines located in the wings of Balmer lines. When restricting the comparison to lines in common, the agreement is much better (Table A.1).

HD 119537 is detected as a spectroscopic binary by Dworetsky (1974). The SB2 nature is clearly visible in our CCFs and they are plotted for both observations in Fig. A.2 together with the labels of the components ("A" being the component with the highest correlation peak). The radial velocities given in Table 2 are the one corresponding to component $\mathrm{A}$ and the difference in radial velocity $(\mathrm{A}-\mathrm{B})$ is $153.4 \mathrm{~km} \mathrm{~s}^{-1}$ in the first observation (2006-06-02) and -140.9 $\mathrm{km} \mathrm{s}^{-1}$ on the second (2012-02-14).
HD 127304 is suspected by Ramella et al. (1989) to be a SB2. Our derived radial velocity is moreover significantly different from the value published by Gontcharov (2006), $-12.6 \mathrm{~km} \mathrm{~s}^{-1}$ (Fig. A.1).

HD 132145 is suspected by Ramella et al. (1989) to be a spectroscopic binary. The observed variation in radial velocity in our data is not significant taking the instrumental offset between both spectrographs into account.

HD 133962 is suspected to be a photometric variable star (Kukarkin et al. 1981).

HD 145647 is suspected to be a binary star from the shape of the CCF (Fig. 2).

HD 145788 is studied by Fossati et al. (2009) who do not detect clear signatures of chemical peculiarity and believe this object is a normal star whose abundance pattern reflects the composition of its progenitor cloud. It is flagged by Ramella et al. (1989) as showing a profile disagreeing with a rotational broadening, probably due to the small rotational broadening. In Fig. A.3g, the shape of the main lobes of the observed profiles agree with the theoretical rotation profile. This star was only observed with ÉLODIE and in our classification, it falls in the normal group. In the sample, it is the normal star with the smallest $v \sin i$.

HD 156653 is indicated as a spectroscopic binary by Dworetsky (1974). Our radial velocity measurements show a ratio of the external error over the internal error just above the threshold used to identify variations in Table 3.

HD 158716 is suspected by Ramella et al. (1989) to be a spectroscopic binary. In our classification, it falls in the CP group and is newly detected as chemically peculiar.

HD 172167 is, strangely enough, an outlier in Fig. A.1, where Gontcharov (2006) gives a radial velocity of $-20.6 \mathrm{~km} \mathrm{~s}^{-1}$. Vega is not known to have a variable radial velocity on such a scale.

HD 174567 is suspected by Ramella et al. (1989) to be a variable CP star (strong $\mathrm{Si}$ and variable $\mathrm{Sr}$ ). It is classified as a normal star according to our measurements but we detect a variation in radial velocity, strengthened by the comparison with Gontcharov (2006) in Fig. A.1.

HD 176984 is suspected to be a spectroscopic binary from the variability of its radial velocity (Table 3 ).

HD 183534 is detected as a spectroscopic binary from the shape of its CCF (Fig. 2) and the difference in radial velocity with Gontcharov (2006) in Fig. A.1.

HD 196724 is suspected to be a spectroscopic binary from the variability of its radial velocity (Table 3 ).

HD 199095 is detected as a spectroscopic binary by Dworetsky (1974). Our measurements show a variation in radial velocity, confirmed when comparing with data from Gontcharov (2006) in Fig. A.1. 
HD 217186 is considered to be a possible magnetic field candidate by Schröder et al. (2008) after being associated with a ROSAT X-ray source (Schröder \& Schmitt 2007) as a bona fide single star. This object is however suspected to be a binary from the shape of its CCF (Fig. 2). We have only one spectrum for this object, but the comparison with Gontcharov (2006), in Fig. A.1, suggests a variation in radial velocity.

HD 219290 is listed as "uncertain" in Table 5 and lies in the "uncertain" zone in Fig. 9. The high abundances in carbon and oxygen produce contradictory classifications when 10 and 14 are used. The remaining pattern ( $\mathrm{Si}, \mathrm{Ca}, \mathrm{Sc}, \mathrm{Cr}, \mathrm{Fe}, \mathrm{Ni}, \mathrm{Sr}$ and $\mathrm{Zr}$ ) is rather similar to a $\mathrm{CP}$ star.
HD 219485 is listed as "probably normal" in Table 5. It is marginally classified as a CP star based on the 10 "classical" elements. The abundances in $\mathrm{Ni}$ and $\mathrm{Sr}$ are however significantly higher than the median values in the normal star pattern displayed in Fig. 10.

HD 223386 is flagged as disagreeing from a rotation profile by Ramella et al. (1989), but the agreement is very good in our data (Fig. A.3h)

HD 223855 is indicated as a spectroscopic binary by Dworetsky (1974). It shows a significant difference between the measurements of $v \sin i$ using spectral synthesis and Fourier profiles, and both values are significantly higher than the one derived by Dworetsky (1974): $45 \mathrm{~km} \mathrm{~s}^{-1}$. 
F. Royer et al.: Normal A0-A1 stars with low rotational velocities. I.

\section{Appendix B: Linelist}

The analyzed spectral lines are listed in Table B.1, sorted by chemical element and central wavelength, together with the adopted oscillator strength.

Table B.1. Atomic data and their reference for the lines used in our abundances determination.

\begin{tabular}{|c|c|c|c|c|c|c|c|c|c|c|c|}
\hline Element & $\lambda(\AA)$ & $\log g f$ & Ref. & Element & $\lambda(\AA)$ & $\log g f$ & Ref. & Element & $\lambda(\AA)$ & $\log g f$ & Ref. \\
\hline $\mathrm{C}_{\mathrm{I}}$ & 4932.049 & -1.658 & 1 & $\mathrm{Sc}_{\text {II }}$ & 4246.822 & 0.242 & 1 & $\mathrm{Ni}$ & 4470.472 & -0.310 & 3 \\
\hline $\mathrm{C}_{\mathrm{I}}$ & 5052.167 & -1.303 & 1 & $\mathrm{Sc}_{\text {II }}$ & 4374.457 & -0.418 & 1 & $\mathrm{Ni}$ I & 4480.561 & -1.491 & 3 \\
\hline $\mathrm{C}_{\mathrm{I}}$ & 5380.337 & -1.616 & 1 & Sc ${ }_{\text {II }}$ & 4670.407 & -0.576 & 1 & $\mathrm{Ni}$ I & 4490.049 & -2.108 & 3 \\
\hline $\mathrm{C}_{\mathrm{I}}$ & 5793.120 & -2.063 & 1 & Sc II & 5031.021 & -0.400 & 1 & $\mathrm{Ni}$ I & 4490.525 & -2.324 & 3 \\
\hline $\mathrm{C}_{\mathrm{I}}$ & 5800.602 & -2.337 & 1 & Sc II & 5239.813 & -0.765 & 1 & $\mathrm{Ni}$ & 4604.982 & -0.250 & 3 \\
\hline $\mathrm{O}_{\mathrm{I}}$ & 5330.726 & -2.416 & 1 & Sc II & 5526.790 & 0.020 & 1 & $\mathrm{Ni}_{\mathrm{I}}$ & 4648.646 & -0.100 & 3 \\
\hline $\mathrm{O}_{\mathrm{I}}$ & 5330.735 & -1.570 & 1 & Sc II & 5657.896 & -0.603 & 1 & $\mathrm{Ni}$ I & 5080.528 & 0.330 & 3 \\
\hline $\mathrm{O}_{\mathrm{I}}$ & 5330.741 & -0.983 & 1 & Ti II & 4163.644 & -0.130 & 4 & $\mathrm{Ni}$ I & 5099.927 & -0.100 & 3 \\
\hline $\mathrm{O}_{\mathrm{I}}$ & 6155.961 & -1.363 & 1 & Ti II & 4287.873 & -1.790 & 4 & $\mathrm{Ni}$ I & 5476.904 & -0.890 & 1 \\
\hline $\mathrm{O}_{\mathrm{I}}$ & 6155.961 & -1.363 & 1 & Ti II & 4417.714 & -1.190 & 4 & Sr II & 4215.520 & -0.169 & 1 \\
\hline $\mathrm{O}_{\mathrm{I}}$ & 6155.971 & -1.011 & 1 & Ti II & 4443.801 & -0.720 & 4 & $\mathrm{Y}_{\text {II }}$ & 4883.684 & 0.070 & 3 \\
\hline $\mathrm{O}_{\mathrm{I}}$ & 6155.989 & -1.120 & 1 & Ti II & 4468.492 & -0.600 & 5 & $Y_{\text {II }}$ & 4900.120 & -0.090 & 3 \\
\hline $\mathrm{O}_{\mathrm{I}}$ & 6156.737 & -1.487 & 1 & Ti II & 4501.270 & -0.770 & 4 & $\mathrm{Y}_{\mathrm{II}}$ & 5087.416 & -0.170 & 3 \\
\hline $\mathrm{O}_{\mathrm{I}}$ & 6156.755 & -0.898 & 1 & $\mathrm{Cr}_{\text {II }}$ & 4592.049 & -1.217 & 5 & $\mathrm{Y}_{\text {II }}$ & 5200.406 & -0.570 & 3 \\
\hline $\mathrm{O}_{\mathrm{I}}$ & 6156.778 & -0.694 & 1 & $\mathrm{Cr}_{\text {II }}$ & 4616.629 & -1.291 & 6 & $\mathrm{Zr}_{\text {II }}$ & 4156.240 & -0.776 & 3 \\
\hline $\mathrm{O}_{\mathrm{I}}$ & 6158.149 & -1.841 & 1 & $\mathrm{Cr}$ II & 4634.070 & -0.990 & 3 & $\mathrm{Zr}$ II & 4161.210 & -0.720 & 3 \\
\hline $\mathrm{O}_{\mathrm{I}}$ & 6158.172 & -0.995 & 1 & $\mathrm{Cr}_{\text {II }}$ & 4812.337 & -1.995 & 3 & $\mathrm{Zr}$ II & 4208.980 & -0.460 & 3 \\
\hline $\mathrm{O}_{\mathrm{I}}$ & 6158.187 & -0.409 & 1 & $\mathrm{Cr}_{\text {II }}$ & 5237.329 & -1.160 & 5 & $\mathrm{Zr}_{\text {II }}$ & 4496.960 & -0.810 & 7 \\
\hline $\mathrm{Mg}_{\text {II }}$ & 4427.994 & -1.201 & 1 & $\mathrm{Cr}_{\text {II }}$ & 5308.440 & -1.810 & 5 & $\mathrm{Ba}_{\text {II }}$ & 4554.029 & 0.163 & 3 \\
\hline $\mathrm{Mg}_{\text {II }}$ & 4481.126 & 0.730 & 2 & $\mathrm{Cr}_{\text {II }}$ & 5313.590 & -1.650 & 5 & $\mathrm{Ba}$ II & 4934.076 & -0.156 & 8 \\
\hline $\mathrm{Mg}_{\text {II }}$ & 4481.150 & -0.570 & 2 & $\mathrm{Fe}_{\text {II }}$ & 4273.326 & -3.258 & 5 & $\mathrm{Ba}_{I I}$ & 6141.713 & -0.810 & 1 \\
\hline $\mathrm{Mg}_{\text {II }}$ & 4481.325 & 0.575 & 2 & $\mathrm{Fe}_{\mathrm{II}}$ & 4296.572 & -3.010 & 5 & & & & \\
\hline $\mathrm{Si}_{\text {II }}$ & 5041.024 & 0.174 & 1 & $\mathrm{Fe}_{\text {II }}$ & 4416.830 & -2.600 & 5 & & & & \\
\hline $\mathrm{Si}$ II & 5055.984 & 0.441 & 1 & $\mathrm{Fe}_{\text {II }}$ & 4491.405 & -2.690 & 5 & & & & \\
\hline Si II & 5056.317 & -0.535 & 1 & $\mathrm{Fe}_{\text {II }}$ & 4508.288 & -2.210 & 5 & & & & \\
\hline Si II & 5466.432 & -0.190 & 3 & $\mathrm{Fe}_{\text {II }}$ & 4515.339 & -2.490 & 5 & & & & \\
\hline Si II & 5688.817 & 0.106 & 1 & $\mathrm{Fe}_{\text {II }}$ & 4520.224 & -2.600 & 5 & & & & \\
\hline Si II & 5978.930 & -0.061 & 1 & $\mathrm{Fe}_{\text {II }}$ & 4522.634 & -2.030 & 5 & & & & \\
\hline $\mathrm{Ca}_{\mathrm{II}}$ & 4489.179 & -0.726 & 3 & $\mathrm{Fe}_{\text {II }}$ & 4541.524 & -3.050 & 5 & & & & \\
\hline $\mathrm{Ca}_{\text {II }}$ & 4489.179 & -2.157 & 3 & $\mathrm{Fe}_{\text {II }}$ & 4555.890 & -2.290 & 5 & & & & \\
\hline $\mathrm{Ca}_{\text {II }}$ & 4489.179 & -0.613 & 3 & $\mathrm{Fe}_{\text {II }}$ & 4582.835 & -3.100 & 5 & & & & \\
\hline Ca II & 5001.479 & -0.517 & 1 & $\mathrm{Fe}_{\text {II }}$ & 4656.981 & -3.630 & 5 & & & & \\
\hline Ca II & 5019.971 & -0.257 & 1 & $\mathrm{Fe}_{\text {II }}$ & 4666.758 & -3.330 & 5 & & & & \\
\hline Ca II & 5021.138 & -1.217 & 1 & $\mathrm{Fe}_{\text {II }}$ & 4923.927 & -1.320 & 5 & & & & \\
\hline Ca II & 5285.266 & -1.153 & 1 & $\mathrm{Fe}_{\text {II }}$ & 5197.577 & -2.100 & 5 & & & & \\
\hline \multirow[t]{2}{*}{ Ca II } & 5307.224 & -0.853 & 1 & $\mathrm{Fe}_{\text {II }}$ & 5276.002 & -1.940 & 5 & & & & \\
\hline & & & & $\mathrm{Fe}_{\text {II }}$ & 5316.615 & -1.850 & 5 & & & & \\
\hline
\end{tabular}

References. (1) NIST; (2) Biermann \& Lübeck (1948); (3) Kurucz (http://kurucz .harvard.edu/LINELISTS/GFALL); (4) Pickering et al. (2002); (5) Fuhr et al. (1988); (6) Sigut \& Landstreet (1990); (7) Biemont et al. (1981); (8) Miles \& Wiese (1969). 